Apidologie, 1970, 1 (3), 233-269.

\title{
DAS MIKROSKOPISCHE BILD EINIGER HONIGE AUS FLORIDA
}

\author{
Le spectre microscopique de quelques miels de Floride
}

Günther VORWOHL

Landesanstalt für Bienenkunde, Stuttgart-Hohenheim

\author{
SUMMARY \\ MICROSCOPICAL SPECTRUM OF SOME FLORIDA HONEYS
}

Florida honeys are characterized by a combination of the following pollens : Ilex, Nyssa, Citrus, Serenoa group. To this combination must be added : Vitis, Rhus-vernix group, Bidens group, Sophora group, Trifolium repens group, Salix, Sambucus niger group, Quercus, Myrica group, Carya, Persea group, Piperaceae/Saururaceae, Itea virginica and Bursera. Ilex, Nyssa, Citrus and pollens of the Serenoa and Rhus vernix groups are predominant. In the 11 true Florida honeys examined, 11 to 15 pollens, out of the above mentioned combination of 18 pollens, have been iden. tified. All pollens found in the samples studied, in so far as they were approximatively identified, are listed in the table. Their frequency of occurence is given. The following pollens are described : Carya tomentosa, Gordonia lasianthus, Fagara rhoifolium, Melia azedarach, Rhus vernix, Ilex glabra, Nyssa ogeche, Richardia scabra, Bidens bipinnata, Helenium tenuifolium, Serenoa repens and Sabal palmetto.

\section{ZUSAMMENFASSUNG}

Die Honige Floridas sind insbesondere ausgezeichnet durch die Pollenkombination Ilex, Nyssa, Citrus, Serenoa-Gruppe. Die Kombination wird ergänzt durch Vitis, Rhus-vernix-Gruppe, BidensGruppe, Sophora-Gruppe, Trifolium repens-Gruppe, Salix, Sambucus niger-Gruppe, Quercus, Myrica-Gruppe, Carya, Persea-Gruppe, Piperaceen/Saururaceen (monocolpat), Itea virginica und Bursera. Ilex, Nyssa, Citrus und Vertreter der Serenoa- und Rhus vernix-Gruppe treten als Leitpollen auf. In den 11 untersuchten authentischen Florida-Honigen wurden jeweils 11-15 Pollen der oben angeführten insgesamt 18 Pollen umfassenden Kombination verifiziert. In einer Tabelle 
sind alle Pollen aufgeführt, die in dem untersuchten Material gefunden wurden, sofern sie wenigstens annähernd identifiziert werden konnten. Ferner wird die Häufigkeit ihres Auftretens angegeben. Die Pollen von Carya tomentosa, Gordonia lasianthus, Fagara rhoifolium, Melia azedarach, Rhus vernix, Ilex glabra, Nyssa ogeche, Richardia scabra, Bidens bipinnata, Helenium tenuifolium, Serenoa repens und Sabal palmetto werden beschrieben.

\section{I. - EINLEITUNG}

Hinsichtlich der Menge des produzierten Honigs steht Florida unter den Staaten der USA zur Zeit an 3. Stelle, während es flächenmäßig erst den 22. Platz belegt. Ein Teil dieser relativ großen Produktion wird exportiert, unter anderem auch in die Bundesrepublik Deutschland, wo er in Form von Mischhonigen, in geringerem Umfang auch als Sortenhonige, in den Handel kommt (z.B. FloridaCitrus-Honig oder Gallberry- (= Ilex Honig). Besonders begehrt im internationalen Handel ist der Tupelo-Honig, der ansprechendes Aroma mit günstigen physikalischen Eigenschaften verbindet. Er bleibt infolge seines hohen Fruchtzuckergehalts unbegrenzt flüssig.

Eingehendere mikroskopische Honiguntersuchungen liegen bisher fast nur aus dem europäischen Bereich vor. Das Pollenspektrum der Honige der wichtigen überseeischen Exportländer ist zwar in groben Zügen bekannt. Im allgemeinen wurden aber diese Kenntnisse an Honigproben gewonnen, die auf den Handelswegen nach Europa kamen, also Proben, deren Authentizität nicht garantiert werden kann. Die Arbeit mit authentischem Material und Proben, deren geographische Herkunft gut lokalisiert ist, bietet erhebliche Vorteile. Die Identifizierung der Pollen wird erleichtert, weil man mit Hilfe von floristischen Handbüchern die Zahl der für das betreffende Gebiet in Frage kommenden Arten, Gattungen und Familien einschränken kann. Eine Differenzialdiagnose zur Bestimmung der geographischen Herkunft des Honigs läßt sich gleichfalls um so leichter und exakter erarbeiten, je genauer die Herkunft des Untersuchungsmaterials bekannt ist.

Dank der freundlichen Vermittlung von Herrn John D. Haynie, Extension apiculturist, University of Florida, Gainesville, erhielt ich im Laufe der letzten Jahre einige authentische Florida-Honige mit Pollen-Vergleichsmaterial, die in der vorliegenden Arbeit ausgewertet werden sollen.

\section{II. - MATERIAL UND METHODEN}

Für die Untersuchung standen 11 authentische Honige zur Verfügung, ferner 2 in der Bundesrepublik aufgekaufte Florida-Orangenhonige. Genauere Angaben sind der Tabelle 1 zu entnehmen. Die Proben stammen aus allen Teilen des Landes, von Miami bis zum Tupelo-Gebiet im Nordwesten.

Die Anfertigung der Honigsedimentpräparate und der Pollenvergleichspräparate erfolgte nach den Vorschriften der Internationalen Kommission für Bienenbotanik (Maurizio und Louveaux, 1962). Zur Frage der Benennung der gefundenen Pollen siehe Vorworl (1968).

Die Zählung der absoluten Teilchenzahl wurde nach dem Verfahren von Louveaux (1961) durchgeführt. 
TAB. 1. -- Liste der untersuchten Honigproben

TABL. 1 - Liste des échantillons de miel examinés

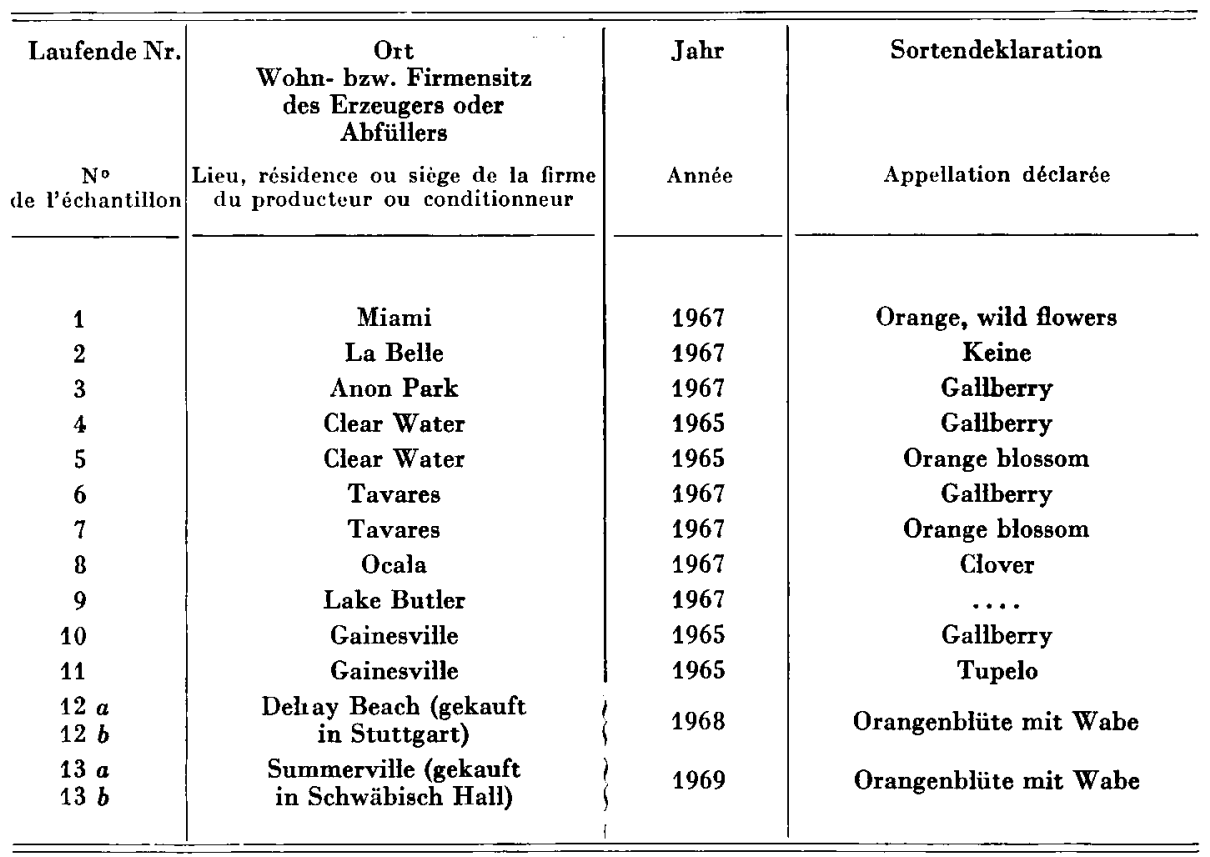

\section{III. - ERGEBNISSE}

\section{1. - Liste der gefundenen Pollenformen}

Im Folgenden werden, nach Familien geordnet, die Pollenformen aufgeführt, die in den Honigen gefunden wurden. Ǔber die Häufigkeit des Auftretens in den einzelnen Honigen unterrichtet die Tabelle 2.

Tав. 2 - Liste der Pollenformen, die in 11 authentischen Florida-Honigen gefunden u'urden und ihre Häufigkeit

$\mathrm{L}=$ Leitpollen (über $45 \%$ ), BP = Begleitpolfen $(16-45 \%), \mathrm{s}=$ wichtiger Einzelpollen $(3-15 \%), v=$ Einzelpollen (unter $3 \%$ der ausgezählten Pollen).

TABL. 2. - Nom et fréquence des types de pollen, trouvés dans 11 miels authentiques de Floride

$\mathrm{L}=$ Pollen dominant (au-dessus de $45 \%$ ), BP = Pollen d'accompagnement (16 à $45 \%$ ), $\mathrm{s}=$ Pollen isolé important (3 à $15 \%$ ), $\mathrm{v}=$ Pollen isolé (au-dessous de $3 \%$ des pollens).

\begin{tabular}{|c|c|c|c|c|c|c|c|c|c|c|c|}
\hline \multirow{2}{*}{$\begin{array}{c}\text { Unterscheidbare Pollenformen } \\
\text { Différents types de pollen }\end{array}$} & \multicolumn{11}{|c|}{$\begin{array}{c}\text { Laufende Nummer des Honigs } \\
\text { Numéro de l'échantillon }\end{array}$} \\
\hline & 1 & 2 & 3 & 4 & 5 & 6 & 7 & 8 & 9 & 10 & 11 \\
\hline Taxaceae/Cupressaceae-Тyp..... & - & - & - & $\mathbf{v}$ & $\mathbf{v}$ & - & $\mathbf{v}$ & - & - & - & - \\
\hline $\begin{array}{l}\text { Pinaceae } \\
\quad \text { Pinus } \ldots \ldots \ldots \ldots \ldots \ldots \ldots\end{array}$ & - & - & - & - & - & - & $\mathbf{v}$ & $\mathrm{v}$ & - & - & - \\
\hline $\begin{array}{l}\text { Myricaceae } \\
\quad \text { Myrica-Gruppe } \ldots \ldots \ldots \ldots\end{array}$ & $\mathbf{s}$ & $\mathbf{v}$ & $\mathbf{v}$ & $\mathbf{v}$ & $\mathbf{v}$ & $\mathbf{v}$ & $\mathbf{v}$ & $\mathbf{v}$ & $\mathbf{v}$ & - & - \\
\hline
\end{tabular}




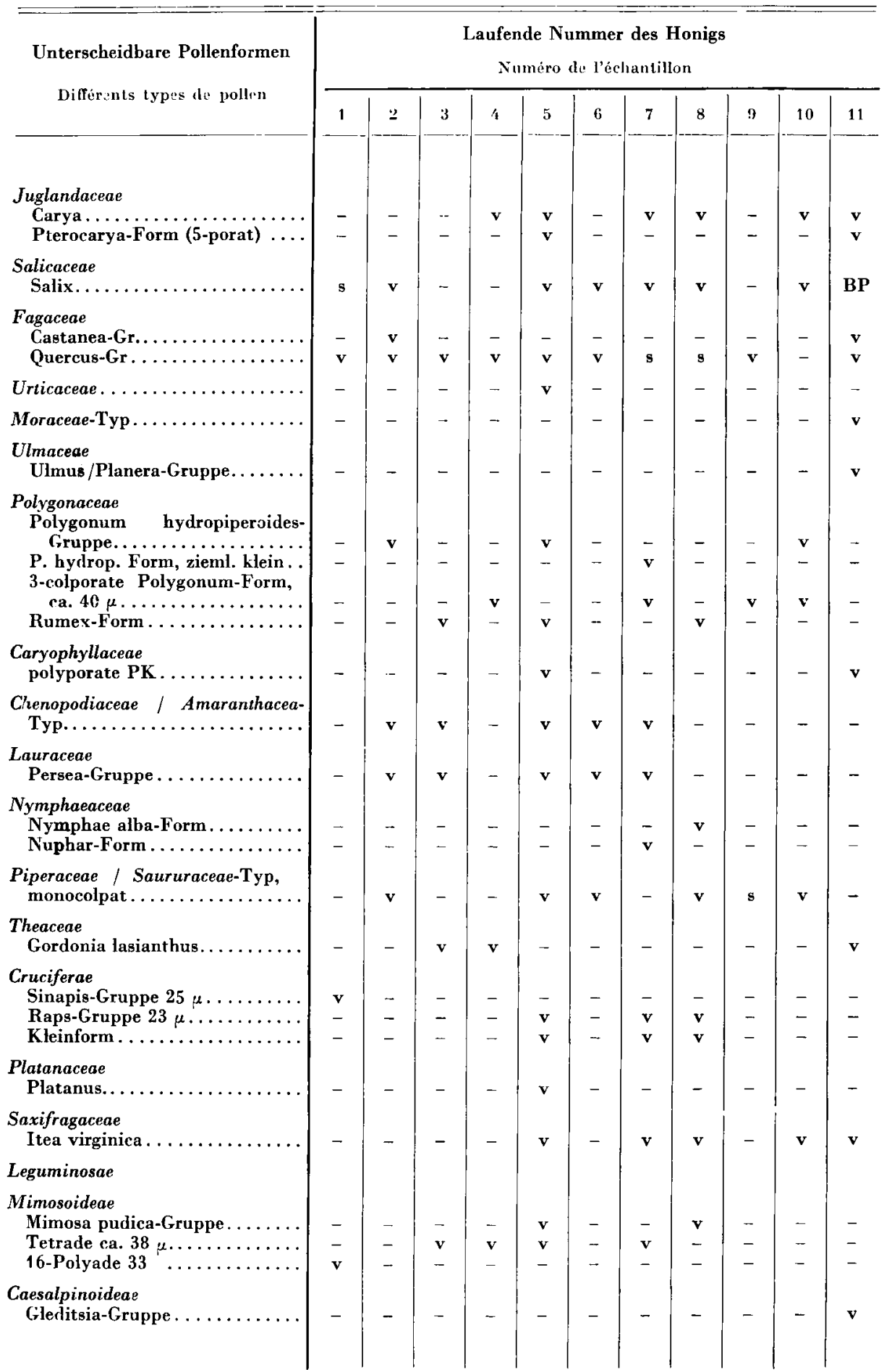


Unterscheidbare Pollenformen

Différents types de pollen

\section{Faboideae}

Trifolium repens-Gruppe

Melilotus-Gruppe

Sophora-Gruppe .

Vicia-Gruppe.

\section{Rosaceae}

Pirus-Gruppe.

Prunus-Gruppe.

\section{Rutaceae}

Citrus

\section{Burseraceae}

Bursera

Meliaceae

Melia azedarach

\section{Anacardiaceae}

Rhus vernix-Form

Pistacia-Typ

\section{Aceraceae}

Acer .

\section{Aquifoliaceae}

$$
\text { Ilex. }
$$

\section{Rhamnaceae}

Rhamnus/Ceanothus-Form

Vitaceae

Ampelopsis / Parthenocissus-

Form ................

Vitis-Gruppe

Vitis-Form.

\section{Cucurbitaceae}

Citrullus.

Myrtaceae.

Cornaceae

Cornus sanguineus-Form

\section{Nyssaceae}

Nyssa.

\section{Umbelliferae}

Umb-Form $\mathrm{H}$

Ericaceae

\section{Rubiaceae}

Galium-Gruppe

\section{Labiatae}

Lab. M-Form

\section{Plantaginaceae}

Plantago.

\section{Caprifoliaceae}

Sambucus nigra-Gruppe

Laufende Nummer des Honigs

Numéro de l'échantillon

\begin{tabular}{|c|c|c|c|c|c|c|c|c|c|c|}
\hline 1 & 2 & 3 & 4 & 5 & 6 & 7 & 8 & 9 & 10 & 11 \\
\hline $\mathbf{v}$ & $\mathbf{v}$ & $\mathbf{v}$ & $\mathbf{v}$ & $\mathbf{v}$ & $v$ & - & $\mathbf{v}$ & $\mathbf{v}$ & - & $\mathbf{v}$ \\
\hline- & - & - & - & $\mathbf{v}$ & $\mathbf{v}$ & - & - & - & - & - \\
\hline $\mathbf{v}$ & $\mathbf{v}$ & BP & $\mathbf{v}$ & $\mathbf{v}$ & $\mathbf{v}$ & $\mathbf{v}$ & $\mathbf{v}$ & $\mathbf{v}$ & $\mathbf{v}$ & $\mathbf{v}$ \\
\hline- & - & - & - & - & - & $\mathbf{v}$ & - & - & - & - \\
\hline- & - & - & - & - & - & - & $\mathbf{v}$ & $T$ & - & - \\
\hline- & - & - & - & - & - & - & - & - & $\mathbf{v}$ & - \\
\hline $\mathbf{s}$ & $\mathbf{v}$ & $\mathbf{v}$ & $\mathbf{v}$ & L & $\mathbf{v}$ & L & s & $\mathbf{s}$ & $\mathbf{v}$ & $\mathbf{v}$ \\
\hline- & $\mathbf{v}$ & - & - & - & - & - & - & - & - & - \\
\hline- & - & - & - & - & $\mathbf{v}$ & $\mathbf{v}$ & - & - & - & - \\
\hline L. & - & $\mathbf{v}$ & $\mathbf{v}$ & $\mathbf{v}$ & $\mathbf{v}$ & $\mathbf{v}$ & - & - & $\mathbf{v}$ & 8 \\
\hline- & - & - & - & - & $\mathbf{v}$ & $\mathbf{v}$ & $\mathbf{v}$ & - & $\mathbf{v}$ & $\nabla$ \\
\hline- & - & - & - & - & - & - & - & - & - & $\mathbf{v}$ \\
\hline $\mathbf{v}$ & B & $\mathbf{s}$ & L & $\mathbf{v}$ & I. & $\mathbf{v}$ & L & I & L & $v$ \\
\hline- & - & - & - & - & - & - & - & - & - & 9 \\
\hline- & $\mathbf{v}$ & - & - & - & $\mathbf{v}$ & - & -- & $\mathbf{v}$ & - & - \\
\hline- & $\mathbf{v}$ & $\mathbf{v}$ & $\mathbf{v}$ & - & $\mathbf{s}$ & $\mathrm{v}$ & $\mathbf{v}$ & $\mathbf{s}$ & $\mathbf{v}$ & $\mathbf{v}$ \\
\hline- & - & $\mathrm{v}$ & $\mathbf{v}$ & - & - & - & - & - & $\mathbf{v}$ & - \\
\hline- & $\mathbf{v}$ & - & $\mathbf{v}$ & $\sim$ & - & - & - & - & - & - \\
\hline- & - & - & - & $\mathbf{v}$ & $\mathbf{v}$ & - & - & - & $\mathbf{v}$ & - \\
\hline $\mathbf{s}$ & $\mathbf{v}$ & $\mathbf{v}$ & $\mathbf{v}$ & - & - & - & - & - & - & - \\
\hline- & $\mathbf{v}$ & - & - & - & -- & $\mathbf{v}$ & - & - & $\mathbf{v}$ & - \\
\hline $\mathbf{v}$ & $\mathbf{v}$ & $\mathbf{v}$ & $\mathbf{v}$ & $\mathbf{v}$ & $\mathbf{v}$ & $\mathbf{v}$ & $\mathbf{v}$ & $\mathbf{v}$ & $\mathbf{v}$ & I. \\
\hline- & - & - & - & - & $\sim$ & $\mathbf{v}$ & - & - & - & - \\
\hline- & - & $\mathbf{v}$ & $\mathbf{v}$ & - & $\mathbf{v}$ & $\mathbf{v}$ & - & - & - & - \\
\hline- & - & - & - & $\mathbf{v}$ & - & - & - & - & - & - \\
\hline- & $\mathbf{v}$ & - & - & - & $\mathbf{v}$ & - & - & - & - & - \\
\hline- & - & - & - & - & $\mathbf{v}$ & - & - & - & - & - \\
\hline $\mathbf{v}$ & $\mathbf{v}$ & $\mathbf{v}$ & $\mathbf{v}$ & $\mathbf{v}$ & $\mathbf{v}$ & $\mathbf{v}$ & $\mathbf{s}$ & $\mathbf{v}$ & $\mathbf{v}$ & $\cdot$ \\
\hline
\end{tabular}




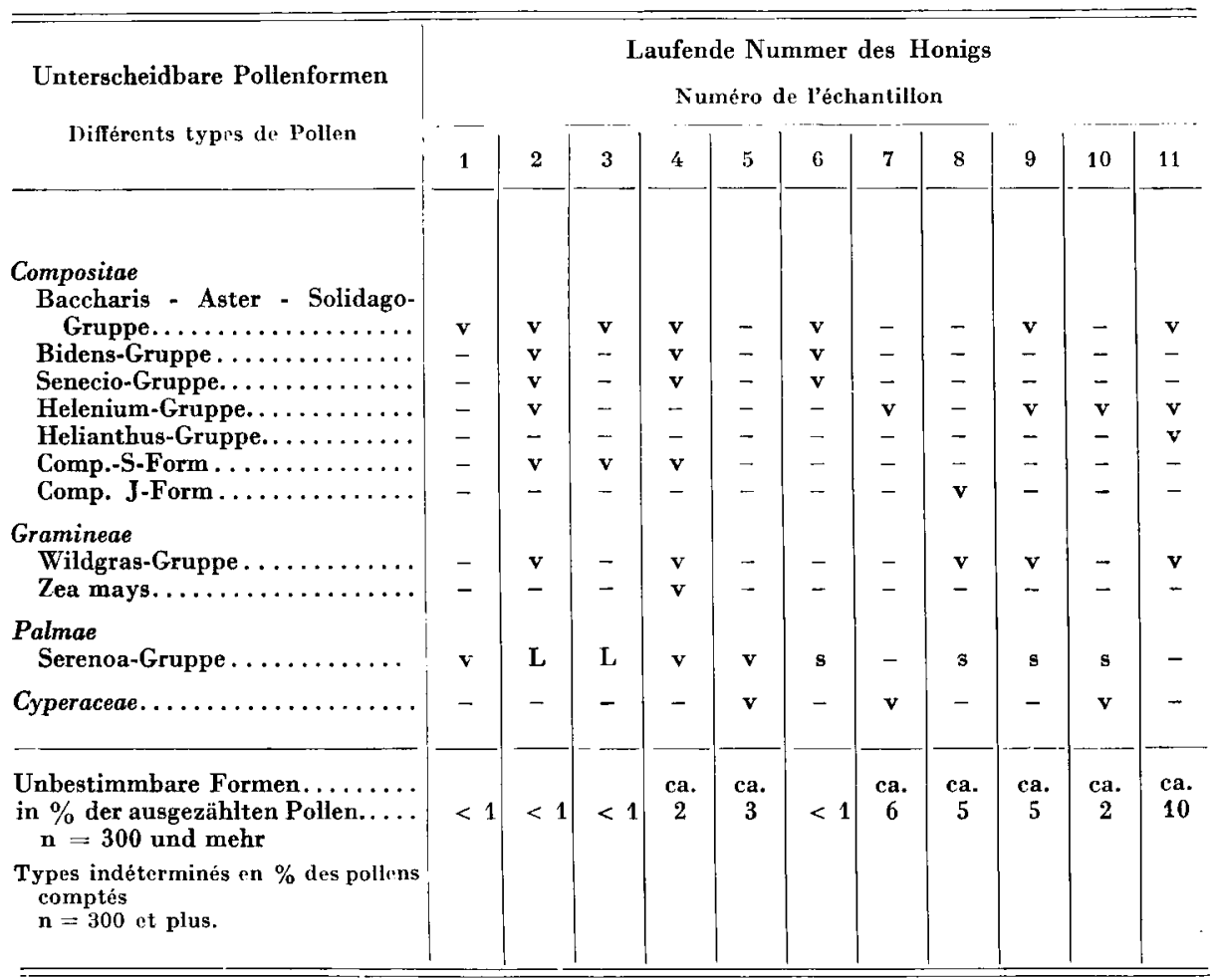

Taxaceen-/Cupressaceen-Typ : Inaperturate sphäroidische PK mit ca. $27 \mu$ Durchmesser aus einer der genannten Familien wurden nur vereinzelt gefunden (die Blüten aller in Frage kommenden Pflanzen sind nektarlos). Beschreibung des Pollens in ZI, G, H, HA, EF1).

Das gleiche gilt von Pinus; das Vorkommen dieses Pollens ist bei den großen Waldungen, die Pinus in Florida bildet, zu erwarten, obwohl diese Conifere keinen Nektar liefert und auch als Pollenquelle wenig bedeutend ist (Beschreibung des Pollens bei ZI, G, H, HA).

Pollen der Myrica-Gruppe (23-25 $\mu$, s. Abb. 1) treten in der Mehrzahl der Honige auf, wenn auch mit geringer Häufigkeit.

Myrica (Cerothamnus) ist in den südöstlichen USA mit mehreren Arten vertreten. Nach ORDETX (1952) liefern die Blüten Nektar. Nach Stirling (zit. nach Ordetx) wird Myrica zu den Trachtpflanzen Floridas gerechnet. Als Vergleichspräparat diente Myrica gale (s. auch HA).

Carya (Abb. 9) wird nach Lovell (1958) auf Pollen beflogen. Sie kommt, wie die meisten Pollen nektarloser Pflanzen, im Honigsediment nur vereinzelt vor. Die Juglandaceen sind ferner mit einem 5 poraten, ca. $40 \mu$ großen PK der Pterocarya-Form vertreten.

Salix kommt in Florida mit zahlreichen Arten vor. Die Bedeutung der Weiden als Bienennährpflanzen ist allgemein bekannt (Beschreibung des Pollens bei ZI, G, H, HA, ML).

In Florida sind mehrere buschförmige Castanea-Arten verbreitet. Sie werden auch von den Bienen beflogen (Peldet, 1922; Oertel, 1939). Die vereinzelt gefundenen PK der Castanea-Gruppe sind etwas kleiner als die von $C$. sativa, die als Vergleich diente (ZI, G, AJ, H, HA, ML, EF).

Die Quercus-Arten liefern zwar keinen Nektar, werden aber gerne auf Pollen beflogen (PELLET, 
1920; OrDETx, 1952; Loveld, 1958). Daher sind sie in geringer Häufigkeit in 10 der 11 untersuchten Honige zu finden. SMall (1933) nennt für Florida 29 Quercus-Arten. Die Abmessungen der Pollen liegen zwischen 23-36 $\mu$. Auf eine Differenzierung zwischen Größenklassen wurde verzichtet (Beschreibung von Quercus-Pollen bei ZI, HA, H).

Triporate PK vom Moracen-Typ (ca. $21 \mu$ ), 5 porate PK der Ulmus/Planera-Gruppe (30 $\mu$ ca.) und 2.3 porate $\mathrm{PK}$ des Urticaceae-Typs (ca. $15, \alpha$ ) wurden nur ganz sporadisch gefunden. (Beschreibungen der Pollen bei ZI, A, G, HA, H.)

Polygonum hydropiperoides wird für Florida als Trachtpflanze genannt (RoBinson, 1956). Pollen dieser Gruppe wurden vereinzelt gefunden. Ferner etwas kleinere Pollen (ca. $27 \mu$ ), die sonst weitgehend $P$. hydropiperoides gleichen (Beschreibung von $P$. hydropiperoides bei E).

Pollen der Rumex-Form (16-27 $\mu$ ) treten vereinzelt auf. Die Pflanzen sind windblütig und nektarlos (ZI, G, HA).

Periporate, sphaeroidische PK von Caryophyllacae (ca. $35 \mu$, Exine gelb) und kleinere des Chenopodiaceae-/Amaranthaceae-Typs spielen im mikroskopischen Bild der untersuchten Honige praktisch keine Rolle. (Beschreibung der erwähnten Formen bei ZI, G, H, HA.)

Recht auffällig, wenn auch nur vereinzelt auftretend, ist ein inaperturates kurzbestacheltes PK (größter Durchmesser ca. $50 \mu, \mathrm{Abb} .2$ ), das große Ähnlichkeit mit Pollen von Laurus nobilis (ZI) hat. Es handelt sich offenbar um Pollen von Persea (s. auch Griebel, 1931, Abb. 315 a). Die Avocadobirne wird in Florida kultiviert und von den Bienen auf Nektar beflogen (Pellet, 1920; Oertel, 1939; Ordetx, 1952). Auch Persea borbonia wird als Trachtpflanze genannt.

Nur in einem Honig wurden vereinzelt Pollen von $N y m p h a e a c e a e$ gefunden, die Ähnlichkeit mit denen von $N$. alba haben (Beschreibung s. Hegi III/3, 1965). In einem Honig kommt sehr vereinzelt Pollen der Nuphar-Gruppe vor (ZI). Nymphaeaceen sind mit mehreren Gattungen in Florida vertreten. Nymphaeaceen gelten als Pollenspender, zum Teil auch als Nektar-Lieferanten (ORDETX, 1952).

In mehreren Honigen wurden kleine monocolpate PK gefunden, (größter Durchmesser 10. $12 \mu$ ). Die Falte ist mit Exineresten besetzt. Im übrigen ist die Exine glatt. Auf den Pollen (Abb. 3) treffen die Beschreibungen zu, die Zander (1941) und Erdtman (1966) für bestimmte Vertreter der Piperaceen und Saururaceen geben. Uther die Bedeutung dieser Familien als Bienennährpflanzen ist mir nichts bekannt.

Als Vertreter der Theaceae wurde vereinzelt der Pollen von Gordonia lasianthus gefunden (Abb. 10). Die Blüten werden beflogen, der Trachtwert der Pflanze ist umstritten (HaynIE, 1966).

PK von Cruciferen kommen in den untersuchten Honigen vergleichsweise außerordentlich selten vor (Beschreibung bei ZI, G, H, HA, EF).

In einem Honig wurden vereinzelt Pollen von Platanus gefunden. Die Platanen sind windblütig und nektarlos (Beschreibung des Pollens s. ZI, HA).

In mehreren Honigen kommt ein charakteristischer diporater Pollen vor (Abb. 4). Nach einer Abbildung bei Enotman (1966) wurde er als Itea virginica (Saxifragaceae) bestimmt. Die Art ist in den südöstlichen USA verbreitet; sie wird in USA und gelegentlich auch in Mitteleuropa kultiviert (van Dersal, 1942; Krüssmann, 1965). Weitere 19 Arten kommen in Ost- und SO-Asien vor (UngleR, 1969). Der größte Durchmesser der in den Honigsedimenten gefundenen Itea-Pollen war $29 \mu$. ErdTMan gibt für acetolysiertes Material $33 \mu$ an. Lovel. (1959) nennt Itea virginica als Trachtpflanze.

1. Für die wichtigsten pollenkundlichen Lehrbücher werden die folgenden Abkürzungen verwendet :

Z I = ZANDER (1935)

Z II = ZANDER (1937).

Z III = ZANDER (1941).

AJ $=$ ARMRRUSTER u. JaCoBs (1934/1935).

G = Griebel $(1930,1931)$.

$\mathrm{H}=$ Hodges (1952).

HA = HYDE u. ADAMs (1958).

ML = MaUnizio u. LouveauX (1965).

EF = Evenius u. Focke (1967).

$\mathrm{E}=$ ErdTMan (1966).

Die näheren bibliographischen Angaben sind im Literaturverzeichnis angegeben. 
Die kleinen Tetraden der Mimosa pudica-Gruppe kommen nur in zwei Honigen vereinzelt vor (Beschr. d. Pollen bei AJ, G, EF). Mimosa pudica kommt im Gebiet vor; nach OndETx wird sie auf Pollen beflogen.

Etwas häufiger ist eine relativ große Tetrade $(39 \mu)$ zu finden (Abb. 5), die gleichfalls den Mimosoideae zuzurechnen ist, aber nicht näher bestimmt werden konnte.

Nur in einem Honig wurde eine 16-Polyade (Acacia-Typ) gefunden (Beschreibung s. ZI, A, G, HA, EF).

Pollen der Gleditsia-Gruppe (ZI) trat nur in einem Honig vereinzelt auf. Für Florida werden von Smal. 2 Gleditsia-Arten (G. aquatica u. G. triacantha) genannt. Pellet (1920) und Ondetx führen Gleditschien als Trachtpflanzen an.

Fast durchgehend kommt in den Honigen vereinzelt Pollen der Trifolium repens-Gruppe vor. Dieser stammt mit einiger Wahrscheinlichkeit wenigstens zum Teil von $T$. repens selbst, der auch in Florida eingebürgert ist. (Beschreibung d. Pollen bei ZI, G, H, ML). Pollen, die denen von Weißklee ähneln, kommen auch bei Vertretern der Gattung Chamaecrista vor. Oentel (1939) nennt unter den Trachtpflanzen Floridas auch $T$. arvense, dessen Pollen gleichfalls zur T. repens-Gruppe gehört. Von T. reflexum und Pollen T. caroliniana, die der gleiche Autor als Trachtquellen Floridas angibt, liegen mir keine Vergleichspräparate vor.

Neben den Pollen der Weißkleegruppe konnten vereinzelt auch Pollen von Melilotus identifiziert werden (ZI, G, H, ML).

Recht regelmäßig wurde in den Honigsedimenten ein sehr kleines $(6-10 \mu)$ tricolporates PK gefunden, dessen Exine undeutlich fein retikuliert und meist reichlich mit gelbem Ö bedeckt ist. Die Pollen haben große Ähnlichkeit mit denen von Sophora japonica und wurden daher als Vertreter der Sophora-Gruppe notiert. Nach SmaLl kommt im Süden Floridas S. tomentosa vor. Ordetx nennt sie als Trachtpflanze (Beschr. d. Pollen von S. japonica bei ZI, AJ).

Pollen der Vicia-Gruppe (ZI, G, H, ML) kommen nur in einem Honig vereinzelt vor. Das gilt auch für die Pollen der Prunus-und Pirus-Gruppe (Stein- und Kernobst) [ZI, G, H, ML, EF].

Citrus-Pollen kommen in allen untersuchten Honigen vor und erreichen in zwei Fällen die Häufigkeit des Leitpollens. Zwischen den verschiedenen Arten und Kulturformen wurde nicht differenziert. Citrus gehört zu den bedeutendsten Honigquellen Floridas.

Pollen der Bursera-Gruppe (Abb. 6, Beschreibung bei E, ferner bei TsukadA, 1964) wurden nur in einem Honig mit einer Häufigkeit von ca. $2 \%$ gefunden. Nach SMALL kommt in Florida nur B. simarouba (= Elaphrium Simaruba) vor und zwar nur im Süden der Halbinsel, woher auch die untersuchte Probe stammt. Man darf daher annehmen, daß auch der Pollen zu dieser Art gehört. Ordetx (1952) nennt $B$. simarouba als Trachtpflanze.

Pelcetr führt den Persischen Flieder Melia azedarach unter den Bienenpflanzen der südostlichen USA an. Der Pollen (Abb. 12) wurde in zwei Honigen vereinzelt gefunden.

Pollen der Rhus vernix.Form (Abb. 13) wurde in der Mehrzahl der Honige gefunden. Rhus. Arten gehören zu den wichtigen Trachtpflanzen der USA (Pellett, 1920; Lovell, 1959b). Als Vergleichspräparate lagen mir Pollen von $R h$. vernix, Rh. typhina und $R h$. copallium vor. Die Pollen der beiden letztgenannten Arten lassen sich von denen des $R h$. vernix unterscheiden. Man muß damit rechnen, daß auch einige Rutaceen Pollen liefern, die der $R h$. vernix-Form ähnlich sind. Als Beispiel führe ich Fagara rhoifolium an (Abb. 11). Die nahe mit Fagara verwandte Gattung Zanthoxyllum (4 Arten in Florida) stellt einige Trachtpflanzen (Pellett, 1920; Oertel, 1939; Ordetx, 1952; Lovell, 1961 a).

Hier ist auch ein Pollen einzuordnen, der nicht näher bestimmt werden konnte. Wegen seiner Ähnlichkeit mit den Pollen von Pistacia wurde er als Pistacia-Typ bezeichnet und unter die Anacardiaceen eingereiht (s. Abb. 7).

Die Pollen von Acer (A. pseudoplatanus-Gr.) wurden nur einmal vereinzelt gefunden (Pollenbeschreibung bei ZI, AJ, HA, ML).

Ilex-Pollen sind in dem untersuchten Material die häufigsten. In der Mehrzahl der Fälle stimmt der Pollen mit denen von 1 . glabra überein. Small nennt für Florida 14 Ilex-Species. Als Trachtpflanzen sind die Ilex-Arten für den Südosten der USA von großer Bedeutung (LovelL, 1956 a).

Pollen der Rhamnus-Ceanothus-Form (ZI, ZIII, AJ, ML, EF) wurden nur in einem Honig gefunden. Rhamnus und Ceanothus und andere Rhamnaceen sind mit zahlreichen Species in Florida vertreten. Viele Rhamnaceen gelten als Trachtpflanzen (Pelletr, 1920; Lovels, 1956 b).

Vitis-Pollen kommt in fast allen untersuchten Honigen vor, als Vergleichspräparat diente 
$V$. vinifera (Beschreibung bei ZI, Z III). Neben den in der Pollage abgerundet dreieckigen Pollen der Vitis-Gruppe kommt auch ein Vitis-ähnliches, aber sphaeroidisches PK vor, das als Vitis-Form bezeichnet wird. Vitis ist in Florida mit zahlreichen Arten vertreten. Sie gelten als Trachtpflanzen (Pellett, 1920; Oertel, 1939).

Nur in zwei Fällen kommen Pollen der Parthenocissus/Ampelopsis-Gruppe vor (ZI, AJ, G, H). Beide Gattungen werden für Florida genannt. PEllet führt sie als Trachtpflanzen an.

Eine Citrullus-Monade konnte nur zweimal identifiziert werden. Das Auftreten des Pollens der Wassermelone wäre zu erwarten, da diese im großnn Umfang in Florida kultiviert wird. Das gleiche gilt für veschiedene Vertreter der Gattung Cucumis (Gurke, Melone). Der Pollen tritt in 2 Honigen vereinzelt auf (Beschreibung von Citrullus bei ZI, G, EF; Beschreibung von Cucumis bei ZI, AJ, G EF).

Die Pollen von Myrtaceen kommen in mehreren Honigen vor. Auf eine Differenzierung in Gruppen nach Größe, Vorhandensein und Größe der Keimhöfe, Syncolpie, usw.) wurde verzichtet. Die Familie ist mit zahlreichen Gattungen und Arten (teils wildwachsend, teils kultiviert) in Florida verbreitet. Sie umfaßt zahlreiche Trachtpflanzen (Ordetx, 1952), Pollenbeschreibung bei ZII, G, AJ, EF.

Pellet führt verschiedene Cornus-Arten als Pflanzen auf, die von Bienen besucht werden, wobei allerdings die in Florida vorkommenden Cornus-(Svida)-Arten nicht genannt werden. In drei der untersuchten Honige waren vereinzeit Pollen der Cornus-Form zu finden, die sich von C. sanguinea, die als Vergleichsmaterial dienten, dadurch unterscheiden, daß sie ausgeprägter prolat sind. (Beschreibung von $C$. sanguinea bzw. $C$. alba bei $\mathrm{ZI}, \mathrm{G}, \mathrm{H}$.)

Die Pollen von Nyssa (AJ, EF) wurden durchgehend in allen Honigen gefunden. Die Gattung ist mit mehreren Arten in Florida vertreten ( $N$. ogeche, $N$. sylvatica, $N$. biflora, $N$. aquatica). Auch die Pollen in den Honigsedimenten variieren etwas in der Ausbildung der Keimstelle und in der Größe. Auf eine Differenzierung wurde verzichtet. Die Nyssa-(= Tupelo) Arten gehören zu den wirtschaftlich bedeutenden Trachtpflanzen Floridas. Uber die besonderen Bedingungen bei Wanderung in das sumpfige Tupelo-Trachtgebiet wurde in der imkerlichen Literatur wiederholt berichtet (Haynie, 1959; Klink, 1960; Niemann, 1936; Rahmlow, 1960).

Die Pollen der Umbelliferen sind in dem untersuchten Material praktisch ohne Bedeutung.

Ericaceen-Pollen-Tetraden wurden vereinzelt gefunden. Es wurde nicht versucht, sie näher zu bestimmen.

Die Rubiaceen sind nur in einem Honig mit Pollen der Galium-Gr. vertreten (ZI, AJ, G, HA). Hingegen wurde Pollen von Richardia scabra (Abb. 14), die als Trachtpflanze für Florida genannt wird (Robinson, 1956; Ordetx, 1952; Oertel, 1939; Pellett, 1920), nicht gefunden. Vereinzeit wurden ähnliche stephanocolpate Pollen gesehen, die möglicherweise auch den Rubiaceen zuzuordnen sind. Vergleichbare Formen kommen auch bei Euphorbiaceen, Polygalaceen und Pedaliaceen (E) vor. Alle genannten Familien sind im Gebiet vertreten.

Die stephanocolpaten (6) PK der Labiaten-M-Form (ZI, AJ, G, H, ML, EF) spiejen im mikroskopischen Bild der untersuchten Honige nur eine geringe Rolle.

Entsprechend der weltweiten Verbreitung der Gattung Plantago finden sich die Pollen dieser Gruppe in Honigen verschiedenster Herkunft. Im vorliegenden Material sind sie nur einmal vereinzelt vertreten. Plantago wird zu den Pflanzen gerechnet, die nur Pollen spenden (Ordetx, 1952).

Erstaunlich regelmäßig (nur einmal fehlend) ist Sambucus-Pollen (der S. niger-Gruppe, s. Abb. 8) in den untersuchten Honigen zu finden, wenn auch in niedrigen Prozentsätzen. Nach SMall bildet $S$. simpsonii besonders im südlichen Florida große Bestände. Ordetx (1952) nennt diese Holunderart als guten Pollenspender (Beschreibung des Pollens von $S$. niger bei ZI, A, J, H, HA).

Compositen-Pollen der Aster-Gruppe (zu der u. a. auch die Pollen von Solidago und verschiedener Baccharis-Arten gehören) erreichen nur geringe Häufigkeit und sind nicht in allen Honigen vertreten (s. die Beschreibungen von Aster bei ZI, G, H, HA, ML). Zum Trachtwert der genannten Gattungen siehe Pellett (1920), Lovell (1961 $b$ ).

Das gleiche gilt von der Bidens-Gruppe (Abb. 15), die weitgehend dem Pollen von Helianthus annuus gleicht, aber kleiner ist als dieser (ca. $24 \mu$ mit Stacheln). Verschiedene Bidens-Arten werden als Trachtpflanzen genannt (PeldetT, 1920; ORdetx, 1952).

Die Senecio-Gruppe HA, die sich von der Aster-Gruppe besonders dadurch unterscheidet, $\mathrm{daB}$ die Basen der Stacheln deutlichere Streifung zeigen, kommt in wenigen Honigen vereinzelt vor.

Auch die Helenium-Gruppe erreicht nur in 4 Honigen eine geringe Häufigkeit. Die Gattung 
Helenium kommt in Florida mit mehreren Arten vor. H. tennifolium (Abb. 16) kann, wo es größere Bestände bildet, bitteren Honig liefern (Pellett, 1920).

Pollen der Helianthus-Gr. (ZI, ML, EF, G) wurde nur in einem Honig gefunden.

Compositen der S-Form, z.B. von Cirsium-Arten (ZI, G, H, ML, HA, EF) sind gleichfalls in den untersuchten Honigen nur wenig verbreitet. (Cirsium nuttali wird von RoBINson als Trachtpflanze Floridas erwähnt.)

Die Pollen der Compositen T-(Taraxacum)-Form sind im mikroskopischen Bild der untersuchten Honige ohne größere Bedeutung. (Beschreibung der Pollenform bei ZI, G, H, HA, ML, EF.)

Die Compositae-I-Form (nach Centaurea iacea) wurde nur einmal gefunden (Pollenbeschreibung ZI, G, H, HA, ML, EF).

Die Wildgräser haben nur als Pollenlieferanten gelegentliche Bedeutung. Sie wurden in mehreren Honigen gefunden. Der wegen seiner Größe näher identifizierbare Pollen von Zea mays konnte nur einmal festgestellt werden. (Pollenbeschreibung der Gramineae ZI, G, H, HA, E; s. auch Bevg, 1961).

Entsprechend der Bedeutung von Serenoa repens als Trachtpflanze (RoBinson, 1956; ORdetx, 1952; Pellett, 1920), kommt der Pollen der Serenoa-Gruppe in fast allen untersuchten Honigen vor. Im Honigsediment ist der Pollen von Sabal palmetto (Abb. 17) nur schwer von Serenoa zu unterscheiden. Auch Sabal palmetto wird zu den wichtigen Trachtpflanzen Floridas gerechnet (Pellett, 1920; Ordetx, 1952).

Die Pollen von Cyperaceen sind gelegentlich in Honigen verschiedenster Herkunft zu finden. Die Sauergräser werden nur auf Pollen beflogen (ZI, G, H, HA).

Der Prozentsatz der Pollenkörner, die in der Tabelle 1 nicht aufgeführt sind, da bei ihnen die Identifizierung nicht möglich oder zu unsicher war, ist gering. Er erreicht maximal $10 \%$. Die Häufigkeit der einzelnen unsicheren oder unbestimmbaren Formen bleibt jeweils unter $3 \%$, also in der Häufigkeitsklasse " vereinzelt ". Bei den nicht identifizierten Pollen handelt es sich vorwiegend um 3-colpate, 3 colporoidate und 3-colporate oblate bis sphaeroidische PK mit glatter oder wenig skulpturierter Exine in der Größenordnung 15-30 Rosaceen stammen. Bei der Verbreitung des 3-colpaten/3-colporaten Pollentyps im Pflanzenreich ist es aber wenig sinnvoll, ohne umfangreiches Vergleichsmaterial nähere Bestimmungen zu versuchen.

Ferner finden sich monocolpate Pollen mit fast glatter Exine in der Größenordnung 30-80 $\mu$ * Es handelt sich wahrscheinlich um Pollen von Palmen (oder Magnoliaceen). Da der monocolpate Typ gleichfalls in vielen Familien auftritt, schien auch hier eine Benennung ohne ausreichende Vergleichssammlung zu riskant.

Als charakteristisch für Florida-Honige darf auf Grund des untersuchten Materials die Kombination Ilex, Nyssa, Citrus, Serenoa-Gr. angesehen werden, wobei jeder der genannten Pollen als Leitpollen auftreten kann. Die Kombination wird ergänzt durch Vitis, Rhus vernix-Gr., Sophora-Gr., Myrica-Gr., BidensGr., Trifolium repens-Gr., Salix, Sambucus und Quercus, die gleichfalls in der Mehrzahl der Honige vorkommen. Ferner sind die Pollen von Carya, der PerseaGr. und der monocolpaten Piperaceen-Saururaceen-Form zu erwähnen, die öfter gefunden wurden. Schließlich müssen hier noch die Pollen von Itea virginica und Bursera wegen ihrer charakteristicshen Form und ihres relativ begrenzten Verbreitungsareals genannt werden.

Nach Engler (1964) gehört das südliche Florida zum karibischen Florengebiet, der Norden zum Florengebiet südliches atlantisches Nordamerika. Auch hinsichtlich des Pollenbilds der Honige ließen sich wahrscheinlich - bei ausreichend großem Untersuchungsmaterial - Unterschiade zwischen den Honigen des südlichen Landesteils und denen herausarbeiten, die nördlich der Citrus-Region erzeugt wurden. Hingegen ist kein wesentlicher Unterschied zwischen den Honigen des nördlichen Florida und denen der im Norden angrenzenden Gebiete zu erwarten. 
Die Unterscheidung von den Honigen Mitteleuropas und denen Canadas (Louveaux, 1965) macht keinerlei Schwierigkeiten. Soweit dies nach dem heutigen Wissensstand beurteilt werden kann, ist die oben beschriebene Kombination auch im Vergleich mit denen anderer tropischer und subtropischer Länder recht charakteristisch.

Die im Einzelhandel in Deutschland aufgekauften Honigproben (Florida Orangenblütenhonige mit Wabe) brachten bei der mikroskopischen Untersuchung das folgende Ergebnis :

Honig 12 a (Wabenstück) : Serenoa-Gr. (Leitpollen), Ilex (wichtiger Einzelpollen), ferner vereinzelt : Myrica-Gr., Quercus, Sophora-Gr., Prunus-Gr. (groß $70 \mu$ ), Vitis, Rhus vernix-Gr., Sambucus, Bidens-Gr., sowie Mimosa pudica-Gr., Acer, Labiate M $(27 \mu)$, Aster-Gr., Helenium Gr., nicht bestimmbare Formen in der Größenordnung von $1 \%$.

Honig $12 b$ enthält extrem wenig Pollen. Identifiziert wurden : Serenoa-Gr., Ilex, Sambucu, Rhus vernix-Gr., Vitis, Sophora-Gr., Bursera, Trifolium repens-Gr., ca. $2 \%$ nicht bestimmbare Formen.

Im Honig Nr. 13 finden sich : Citrus, Leitpollen (ca. $68 \%$ ), als wichtige Einzelpollen Ilex und Nyssa, ferner vereinzelt Myrica-Gr., Sophora-Gr., Bidens-Gr., Quercus, Rhus vernix-Gr., Trifolium repens-Gr., Persea-Gr., Serenoa-Gr., Sambucus-Gr., Vitis, Carya, Salix und Piperaceen/Saururaceen, Pinus, Aster-Gr., Taxaceen/Juniperaceen-Typ, Umbelliferen H, Vicia-Gr., Wildgräser und Melia azedarach, sowie Betula, Alnus, Richardia Taxodium und Raphanus-Gr.; etwa $7 \%$ der Pollen konnten nicht identifiziert werden. Der Honig aus dem Glas und der Honig aus dem Wabenstück gleichen sich weitgehend und wurden gemeinsam ausgewertet.

Von der oben aufgestellten 18 Pollengruppen umfassenden, für FloridaHonige typischen Kombination finden sich in den authentischen Honigen (Nr.111) jeweils 11-15 Vertreter. Im Honig 12 lassen sich 11 Pollen der charakteristischen Kombination verifizieren, im Honig 13 sogar 16. Die Mehrzahl der sonstigen Pollen, die für die Proben 12 und 13 angeführt werden, treten auch im authentischen Material auf. Darüber hinaus wurden im Honig 13 noch Richardia und Taxodium, Alnus und Betula identifiziert, die im authentischen Material nicht vorkommen. Das Auftreten von Richardia ist in Florida-Honigen zu erwarten. Taxodium distichum bildet im Gebiet große Bestände; es liegt also nahe, da $\beta$ der Pollen sich gelegentlich im Honig findet, obwohl die Taxodien windblïtig sind. Betula und Alnus kommen im Norden des Staates vor. Die Pollenspektren der authentischen Proben und die der beiden in der Bundesrepublik aufgekauften "Florida "-Honige stimmen also sehr gut überein. Es besteht daher kein Zweifel an der Richtigkeit der angegebenen geographischen Deklaration.

\section{2. - Messung des absoluten Gehalts des Honigs an Pollen und sonstigen pflanzlichen Elementen}

Die Resultate der Zählung des absoluten Gehalts an pflanzlichen Elementen sind in Tabelle 3 niedergelegt. MAurizio (1949) hat die Honige nach ihrem Gehalt an pflanzlichen Elementen in Kategorien eingeteilt :

Gruppe I mit weniger als 20.000 pflanzlichen Elementen in $10 \mathrm{~g}$ Honig,

Gruppe II mit 20.000-100.000 Elementen,

Gruppe III mit 100.000-500.000 Elementen in $10 \mathrm{~g}$ Honig und so fort. Die Mehrzahl der von Maurizio untersuchten Schleuderhonige fielen in die Kategorie II. 
TAB. 3 - Absoluter Pollengehalt in Florida-Honigen.

TABL. 3. - Teneur absolue en pollen des miels de Floride

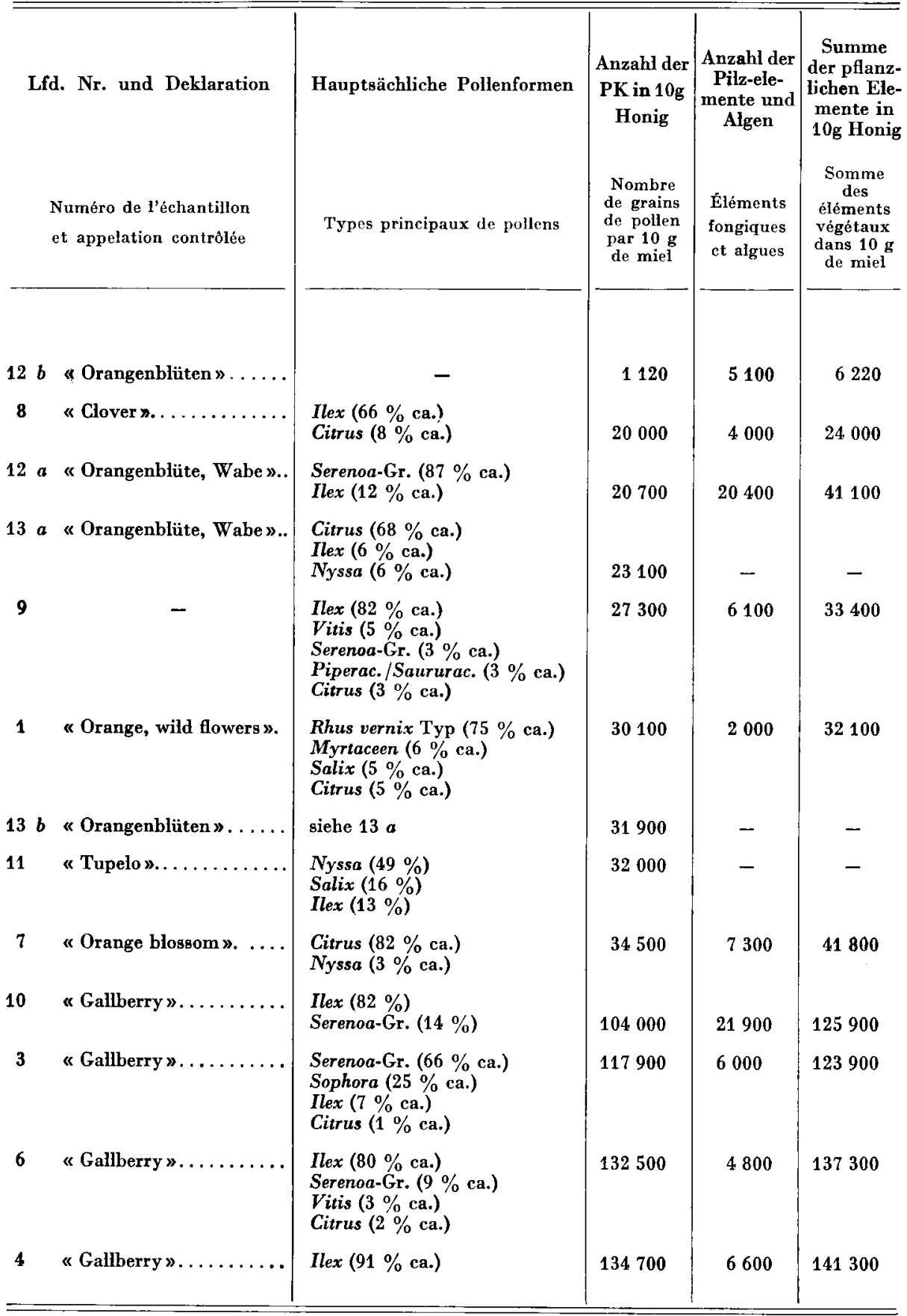


Bei den untersuchten Florida-Honigen fällt die Nr. $12 b$ durch extreme Pollenarmut auf. Es handelt sich offenbar um einen gefilterten Honig. Dafür spricht auch das vereinzelte Auftreten von Partikeln aus Diatomeenerde.

Die Citrus-Honige stellen bekanntlich im Rahmen der mikroskopischen Honiguntersuchung besondere Probleme (Maurizio, 1958). Es gibt Honige, die nach den Umständen ihrer Entstehung Citrus-Honige sein müssen, aber nur einen niedrigen Prozentsatz Citrus-Pollen enthalten. Auch die absolute Zahl der pflanzichen Elemente ist in diesen Honigen gering. Daneben gibt es auch Honige, die Citrus als Leitpollen enthalten und bei denen die absolute Zahl der pflanzlichen Elemente pro $10 \mathrm{~g}$ Honig in die Kategorie II fällt. Die hier untersuchten Citrus-Honige verhalten sich hinsichtlich ihres Pollengehalts " normal ". Der prozentuale Anteil der Citrus-Pollen ist sehr hoch (bei Honig Nr. 13 ca. $68 \%$, bei Nr. 7 ca. $82 \%$ Citrus-Pollen, bei Nr. 5, 72\%). Bei der Nr. 5 reichte die Honigmenge für die Messung der absoluten Teilchenzahl nicht aus.

Die Gallberry Honige (Nr. 4, 6, 10) sind pollenreich und gehören in den unteren Bereich der Gruppe III. Das spricht dafür, daß Ilex zu den im Honig überrepräsentierten Pollen gehört. Honig 9 und 8 , in denen Ilex-Pollen mit ca. 82 respektive $66 \%$ dominiert, zeigen allerdings einen relativ niedrigen Pollengehalt im unteren Bereich der Gruppe II. Das widerspricht nicht unbedingt der Feststellung, der Ilex-Pollen sei leicht überrepräsentiert und der Honig entsprechend pollenreich. Die Honige 8 und 9 enthalten Citrus in der Größenordnung des " wichtigen Einzelpollens " (3-15\%). Ein Anteil von relativ pollenarmem Citrushonig könnte den niedrigen Pollengehalt der beiden in Frage stehenden Proben erklären.

Honig Nr. 3 mit Serenoa als Leitpollen fällt in die Kategorie III. Die Probe 2 wurde ganz für andere Zwecke verbraucht, sodaß kein Honig für die Messung der absoluten Teilchenzahl übrig blieb. Nach der Pollendichte im mikroskopischen Präparat dürfte der Honig als pollenreich anzusprechen sein. Hingegen enthält die Honigprobe $12 a$, die direkt der Wabe entnommen wurde und die gleichfalls Serenoa als Leitpollen führt, nur eine " normale " Pollenmenge, was mit der Gewinnungart zusammenhängen könnte, die die sekundäre Anreicherung des Honigs mit Pollen bei der Entdeckelung und Schleuderung ausschließt.

Bei der geringen Zahl der untersuchten Honigproben lassen sich verständlicherweise keine endgültigen Aussagen über den Repräsentationsgrad der behandelten Pollen machen. Die hier gegebenen Erläuterungen können nur dazu beitragen, die Fragen aufzuzeigen, die durch ergänzende Untersuchungen gelöst werden müssen.

\section{3. - Sortendiagnosen}

Die Sortendeklarationen, die den untersuchten Honigproben gegeben wurden, werden in der Mehrzahl der Fälle durch den mikroskopischen Befund bestätigt.

Der Honig Nr. 3 ist allerdings nach dem mikroskopischen Bild nicht als Gallberry sondern als Serenoa-Honig anzusprechen. Im Honig Nr. 8 finden sich nur vereinzelt Kleepollen. Pollenanalytisch muß man ihn als Ilex-Honig bezeichnen.

Interessant ist der Honig Nr. 11. Nyssa stellt in Übereinstimmung mit der Deklaration den Leitpollen. Daneben enthält das Sediment aber sehr viel Pilzelemente (Hyphen und Sporen). Das Zahlenverhältnis der Pollen zu den sogenannten Honigtauelementen liegt bei 1 : 5 . Nach den üblichen Auswertungsregeln müßte es sich also um einen Honigtauhonig handeln. Dem widerspricht jedoch der Sinnenbefund und das Ergebnis der Messung der elektrischen Leitfähigkeit (VoRwoHL,

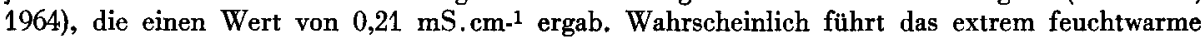
Klima der Tupelo-Region zu einem sehr üppigen Wachstum epiphytischer Pilze, sodaß mit geringfügigen Honigtaumengen oder schon durch Kontaminierung des Nektars und der Bienen große Mengen von Pilzelementen auch in den Blütenhonig gelangen können.

Der Honig $12 a$ ist nach dem mikroskopischen Befund ein Serenoa-Honig. Citrus-Pollen konnte, auch vereinzelt, nicht nachgewiesen werden.

Auf den Honig $12 b$ können die Regeln der Sortendiagnose aus den oben erwähnten Gründen nicht angewendet werden. 


\section{IV. - BESCHREIBUNG DER POLLEN EINIGER TRACHTPFLANZEN FLORIDAS}

1. - Carya tomentosa Nutt. (= C. alba K. Koch)

Juglandaceae, Juglandales

Pollenprobe und Präparat Hohenheim, 1968, Abb. 9.

Populärname englisch : Mockernut.

Verbreitung : östliche U.S.A., Florida bis Texas, nördlich bis Nebraska, Ontario und Vermont. Als Parkbaum gelegentlich in Europa.

Ziemlich große, isopolare PK von hellgrüner Farbe. Pollagen circulär bis semiangulär; Seitenlagen oval. 3 (4) runde bis ovale Poren, maximaler Porendurchmesser $3 \mu$. Exine $2 \mu$, um die Poren etwas dicker, intectat, psilat, innen rauh. Intine unterschiedlich dick, unter den Poren verstärkt, vielfach mit Knitterzeichnung. Cytoplasma mit zahlreichen unregelmäßigen Vakuolen.

$$
\mathrm{P}=38 \mu, \quad \mathrm{A}=45 \mu, \quad \mathrm{P} / \mathrm{A}=0,83 \quad(\mathrm{n}=50) .
$$

\section{2. - Gordonia lasianthus (L) Ellis, Theaceae, Guttiferales}

Pollenprobe : Gainesville, Fla. 1966, Präparat Hohenheim 1966, Abb. 10.

Populärnamen : engl. Loblolly bay, Black laurel.

Verbreitung : östl. U.S.A., Florida bis Louisianna u. North Carolina. Große, gelbe, isopolare Pollenkörner. 3 Colpi, Breite der Falten am Aquator ca. $15 \mu$, Polfeld klein, Abstand der Faltenenden voneinander $10 \mu$, Faltenränder unscharf, gerade, Falten spitz zulaufend. Exine $1,5 \mu$, tectat, Columellen deutlich, reticuliert, Durchmesser der Brochi maximal 1,5 $\mu$. Das Reticulum wird nach den Faltenrändern zu feiner. Intine dünn unter $1 \mu$. Cytoplasma fein granuliert. $\mathbf{P}=42 \mu$, $\mathrm{A}=55 \mu(\mathrm{n}=50), \mathrm{P} / \mathrm{A} 0,76$.

\section{3. - Fagara rhoifolium Engler (=- Zanthoxyllum rhoifolium Lam.) Rutaceae, Rutales}

Pollenprobe Santa Maria*, Rio Grande do Sul, Brasilien, Präparat Hohenheim 1967, Abb. 11.

Verbreitung : Brasilien.

Kleines gelbes bis bräunlich gelbes isopolares PK. Polansicht circulär bis semiangulär, Seitenansicht oval. 3 Porenfalten, Länge ca. $12 \mu$, Pore rund, $2 \mu$ Durchmesser, Exine ca. $1 \mu$ dick, reticuliert, Brochi maximal $1 \mu$ im Durchmesser. Intine $0,7 \mu$, um die Poren verdickt bis auf $1,7 \mu . P=17 \mu, A=16 \mu(\mathrm{n}=70)$, $\mathrm{P} / \mathrm{A}=1,1$.

\section{4. - Melia azedarach, Meliaceae, Rutales}

Pollenprobe Santa Maria, Brasilien, 1966, Präparat Hohenheim 1967, Abb. 12

Populärnamen : deutsch : Persischer Flieder, Syrischer Holunder; englisch : China berry, China tree, Pride of India; französisch : Lilas Margousier, Lilaє, Azèdarach; spanisch : Paraiso, Lila, Lilaila.

* Die Pollenprobe wurde mir freundlicherweise zusammen mit Pollen anderer brasilianischer Trachtpflanzen von Herrn Prof. B. Schirmer, Santa Maria, zugesandt. 
Heimat : Asien, in allen wärmeren Gegenden kultiviert; in den U.S.A. von Florida bis Texas, Oklahoma und North Carolina.

Mittelgroßes, isopolares, blaß grau-gelbes PK. Polansicht tetrangulär, Seitenansicht oval. 4 Porenfalten, Abstand der Faltenenden voneinander 14-18 $\mu$, Poren rund, Faltenränder gerade, an den Poren etwas aufgewölbt und rauh. Breite der Falte und Durchmesser der Pore 4-6 $\mu$. Exine 0,7-0,8 $\mu$, scabrat, an den Poren etwas verdickt. Intine derb, 2,2-2,3 $\mu$. Cytoplasma oft mit Leucoplasten. $P=35 \mu$, $\mathrm{A}=40 \mu$ (gemessen von Intercolpium zu Intercolpium), $\mathbf{n}=50, \mathrm{P} / \mathrm{A}=0,88$.

\section{5. - Rhus vernix L. Anacardiaceae, Sapindales}

Pollenprobe und Präparat Hohenheim 1968, Abb. 13.

Populärnamen : deutsch : Giftsumach; englisch : Poison sumac, Swamp sumac, Thunderwood.

Verbreitung : Östliche U.S.A., Florida bis Louisiana, Minnesota u. Maine.

Ziemlich kleines, blaß grünlich gelbes isopolares PK. Polansicht semiangulär bis circulär, Seitenansicht circulär — breit oval. 3 Porenfalten, Äbstand der Faltenenden voneinander 5-6 $\mu$, Breite der Porenfalte am Äquator $6 \mu$, Faltenbegrenzung wenig deutlich, gerade, Faltenenden spitz. Exopore rundlich, Durchmesser ca. $6 \mu$, Endopore oval (großte Achse in äquatorialer Richtung). Exine ca. 1,5 dick, striat-reticulat, Brochi maximal $1 \mu$, Intine dünn $0,5 \mu$, gelbes $0 ̈ \mathrm{E}, \mathrm{P}=$ $23 \mu, \mathrm{A}=26 \mu, \mathrm{P} / \mathrm{A}=0,9(\mathrm{n}=50)$.

\section{6. - Ilex glabra (L) Gray, Aquifoliaceae, Celastrales}

Pollenprobe : Gainesville 1966, Präparat Hohenheim, 1966.

Populärnamen : deutsch : Tintenbeere, engl. : Gallberry, Evergreen winterberry, franz. : Houx.

Verbreitung : Florida bis Louisiana und Nova Scotia (Canada).

Ziemlich kleines bis mittelgroßes, blaßgelbes, isopolares PK. Polansicht circulär, Seitenansicht circulär-breit oval. 3 Porenfalten, Abstand der Faltenenden voneinander ca. $9 \mu$, Durchmesser der Poren 6-7,5 $\mu$. Exine $3 \mu$ dick (inclusive der Skulpturelemente), gemmat. Gemmen maximal $2 \mu$ hoch. Durchmesser in der Aufsicht gleichfalls $2 \mu$. Nach den Faltenrändern zu werden die Gemmae kleiner. Intine $1-1,5 \mu, \mathrm{P}=30 \mu, \mathrm{A}=31 \mu(\mathrm{n}=50), \mathrm{P} / \mathrm{A}=0,95$.

\section{7. - Nyssa ogeche March, Nyssaceae, Umbelliflorae}

Pollenprobe : Gainesville 1966, Präparat Hohenheim 1966.

Populärnamen : deutsch : Tupelobaum, engl. : Tupelo gum tree, Ogeche lime, franz. : Nysse, Tupélo.

Verbreitung : Florida bis South Carolina.

Ziemlich große bis mittelgroße blaßgraue PK. Polansicht semiangulär, Seitenansicht breit oval. 3 schmale Porenfalten, Abstand der Faltenenden voneinander 10-15 $\mu$, Faltenränder gerade, spitz zulaufend, Exopore rundlich, Durchmesser $3 \mu$, Endopore oval, größter Durchmesser (in äquatorialer Richtung) 4,5 $\mu$, Exine 1,5 $\mu$, an Poren verdickt (auf $3 \mu$ ), keine deutlichen Columellen, scabrat. Intine $1 \mu$, an den Poren verdickt. $\mathrm{P}=32 \mu, \mathrm{A}=41 \mu(\mathrm{n}=30)$. 


\section{8. - Richardia scabra St. Hil. Rubiaceae, Gentianales}

Pollenprobe : Gainesville 1967, Präparat Hohenheim 1967, Abb. 14.

Populärnamen : engl. : Mexican clover, span. Richardia.

Verbreitung : Florida bis Texas, North Carolina und Indiana, Mexico, Mittel und Südamerika, Antillen.

Große, isopolare, radiarsymmetrische, hellgraue PK. Polansicht circulär, Seitenansicht oval. 10-13 Colpi, Länge 10-12 $\mu$, Breite 5-6 $\mu$, Abstand zwischen gegenüberliegenden Falten ca. $40 \mu$, Breite der Intercolpien am Äquator 10-11 $\mu$. Exine ca. $2 \mu$, semitectat, Columellen bis zu $2 \mu$ dick, Intine ca. $1 \mu$ dick.

$\mathrm{A}=54 \mu(\mathrm{n}=20), \mathrm{P}=38 \mu(\mathrm{n}=5), \mathrm{P} / \mathrm{A}=$ ca. 0,88 .

\section{9. - Bidens bipinnata L. Compositae (Unterfamilie Asteroideae, Tribus Heliantheae) Campanulales}

Pollenprobe : Gainesville 1967, Präparat Hohenheim 1967, Abb. 15.

Populärnamen : deutsch : Zweizahn, engl. : Spanish needle, span. : Romerillo.

Verbreitung : Florida bis Arizona, Nebraska und Rhode Island, Mexico, Mittelamerika und Südamerika.

Ziemlich kleine, isopolare, blaß grau-gelbe PK. In der Polansicht circulär, in der Seitenansicht circular bis breit oval. 3 Porenfalten, Länge der Falte $7.8 \mu$, Faltenränder undeutlich, Durchmesser der Exopore 3-4 $\mu$. Ectexine und Endexine (ohne Stacheln) zusammen etwa $1 \mu$. Ectexine mit Stacheln von 2,5 $\mu$ Länge. Die Stachelbasen erscheinen in der Seitenansicht gestrichelt. Intine dünn 0,5 .

$\mathrm{P}$ (mit Stacheln) $24 \mu$, ohne Stacheln $19 \mu(\mathrm{n}=10), \mathrm{P} / \mathrm{A}$ (ohne Stacheln) 0,97 .

10. - Helenium tenuifolium Nutt. Compositae (Unterfam. Asteroideae, Tribus Helenieae), Campanulales

Pollenprobe Baton Rouge, Louisiana, 1967, Präparat Hohenheim 1967, Abb. 16.

Vulgärnamen : deutsch : Dünnblättrige Sonnenbraut, engl. Bitterweed, span. : Pinito.

Verbreitung : Florida bis Texas, Montana und Virginia, Mexico.

Ziemlich kleines, isopolares, blaßgraues PK. Polansicht circulär, Seitenansicht circulär bis breitoval. 3 Porenfalten, Breite 3-4 $\mu$, Länge ca. $10 \mu$, Faltenränder gerade, Falten spitz zulaufend, Pore rundlich, Durchmesser 3-4 $\mu$, Ectexine und Endexine zusammen etwa 1,5 $\mu$. Ectexine mit derben Stacheln, Länge $4 \mu$, Breite an der Basis 4,5 $\mu$. Stacheln mit deutlicher Strichelzeichnung. Intine $0,5 \mu$ dick. P (mit Stacheln) $26 \mu$, ohne Stacheln $18 \mu$, A (mit Stacheln) $27 \mu$, ohne Stacheln $19 \mu, \mathrm{P} / \mathrm{A}$ (ohne Stacheln) 0,95.

\section{1. - Sabal palmetto (Walt.) Todd. Palmae, Principes}

Pollenprobe : La Belle, Fla., 1967, Präparat Hohenheim 1967, Abb. 17. Vulgärnamen : deutsch : Palmettopalme, engl. : Cabagge palm, Cabagge palmetto. 
Verbreitung : Florida, North Carolina, Cuba, Bahamas.

Mittelgroßes, isopolares, blaß graues PK. In der Polansicht unregelmäßig circulär, in den Seitenlagen unregelmäßig oval bis abgerundet vierseitig. Eine große Falte über die ganze Länge des Pollenkorns, bei Aufsicht auf die Falte deutlich begrenzt. Faltenumriß oval, Faltenbreite ziemlich variabel in der Größenordnung von $25 \mu$. Ectexine und Endexine zusammen 1,5 $\mu$, nach den Falten zu dünner werdend, reticulat, Lumina rundlich, Durchmesser maximal $1 \mu$, Dicke der Muri entspricht oder übersteigt den Durchmesser der Lumina; kurze undeutliche Columellen. Intine von wechselnder Dicke.

$\mathbf{P}=32 \mu(\mathbf{n}=100), \mathbf{A}_{1}:$ (von der Mitte des Intercolpiums zur Mittellinie des Colpus) $=31 \mu(\mathrm{n}=25) ; A_{2}$ : (vom Rand des Intercolpiums zum gegenüberliegenden Intercolpienrand) $29 \mu(\mathrm{n}=10)$.

\section{2. - Serenoa repens (Bartr.) Small, Palmae, Principes}

Pollenprobe : Gainesville 1966, Präparat Hohenheim 1966.

Vulgärname : deutsch : Sägefächerpalme, engl. : Saw palmetto, Verbreitung : Florida bis Louisiana und South Carolina.

Die PK gleichen denen von Sabal palmetto, sind aber etwas kleiner. Die Exine erscheint im optischen Schnitt infolge der stärker entwickelten Columellae deutlicher quergestreift.

Für die Erledigung der Dunkelkammerarbeiten bei der Herstellung der Abbildungen bin ich Herrn Th. Held zu Dank verpflichtet.

Eingegangen im Juni 1969.

Reçu pour publication en juin 1969.

\section{RESUME}

Les miels de Floride se caractérisent, en particulier, par la combinaison pollinique des groupes Ilex, Nyssa, Citrus, Serenoa. Cette combinaison est complétée par les pollens de Vitis, des groupes Rhus vernix, Bidens, Sophora, Trifolium, de Salix, du groupe Sambucus nigra, de Quercus, du groupe Myrica, de Carya et de Persea, des Piperaceae-Saururaceae monocolpés, de Itea virginica et de Bursera.

Les pollens dominants sont Ilex, Nyssa, Citrus et ceux du groupe Rhus vernix.

Les figures 1 à 8 représentent ces pollens sur des photos de sédiments de miel, à l'exception des pollens généralement connus de Salix, Vitis et $T$. repens et du genre Carya, décrits ultérieurement.

Dans les 11 miels de Floride authentiques examinés, on a identifié chaque fois 11 à 15 de l'ensemble des 18 pollens mentionnés ci-dessus.

Dans le tableau 2 sont cités tous les pollens trouvés dans le matériel étudié, dans la mesure où ils ont pu être au moins approximativement identifiés. Leur fréquence d'apparition est indiquée à la suite. Dans les notes concernant la liste des pollens (chap. III, $\$ 1$ ), on a indiqué où les pollens en question sont décrits dans la littérature.

Le chapitre IV donne la description des pollens de Carya tomentosa, Gordonia lasianthus, Fagara rhoifolia, Melia azedarach, Rhus vernix, Richardia scabra, Bidens bipinnata, Helenium tenuifolium, Sabal palmetto, Serenoa repens, Ilex glabra et Nyssa ogeche, avec des figures détaillées, sauf pour les 3 derniers cités ( $\mathbf{n}^{\circ} 9$ à 17). 


\section{LITERATUR}

Armbruster L. u. Jacobs J., 1934-1935. Pollenformen u. Honigherkunftsbestimmung. Arch. Bienenkde, 15-16, 277-308, 17-106.

Beug H. J., 1961. Leitfaden der Pollenbestimmung, Lfg. 1. Gustav Fischer, Stuttgart.

Van Dersal W. R., 1942. Ornamental American Shrubs, Oxford University Press, New York, London, Toronto.

Engler A., 1964. Syllabus der Pfanzenfamilien, BD. II. Borntraeger, Berlin.

Erdtman G., 1966. Pollen morphology and plant taxonomy, Hafner Publishing Company, New York and London.

Evenius J. u. Focke Elida, 1967. Mikroskopische Untersuchung des Honigs. In : Handbuch der Lebensmittelchemie, 5. Bd. 1. Teil Springer, Berlin, Heidelberg, New York.

Griebel C., 1930. a. Zur Pollenanalyse des Honigs. I. Mitt Z. Unters. Lebensmittel, 59, 63-79.

Grieber C., 1930-1931. b. Zur mikroskopischen Pollenanalyse des Honigs II-IV Mitt. Z. Unters. Lebensmittel, 59 197-211, 59, 441-471, 61, 241-306.

HaYnIE J. D., 1959. Florida beekeeping on the move. Amer. Bee J., (11), 454-455.

HAYNIE J. D., 1966. Briefliche Mitteilung.

Hegr, 1965. Illustrierte Flora von Mitteleuropa, Bd. 3, Teil 3, 2. Aufl. Hansen-Verl. München. Hodges Dorothy, 1952. The pollen loads of the honey bee, Bee Research Association, London. Hyde H. A. and Adams K. F., 1958. An atlas of airborne pollen grains, London, Macmillan.

KLINK, 1960. Der Tupelohonig, Sïdwestdeutscher Imker, 12, (10), 310.

Krüssmann G., 1965. Die Laubgehölze. Parey, Berlin u. Hamburg.

Louveaux J., 1961. Verbesserte Techniken der Pollenantyse des Honigs. Z. Bienenforsch, 5, (7), 199-204.

Louveaux J., 1966. Pollenanalyse einiger kanadischer Honige Z. Bienenforsch, 8, (5).

Louveaux J., Maurizio Anna, 1963. Méthodes d'Analyse pollinique des miels. Ann. Abeille,6, (1), 75-76.

Lovell H. B., 1956. a. Let's talk about honey plants, Glean. Bee Culture, 84, (3), 173 à 188.

Lovell H. B., 1956. b. Let's talk about honey plants, Glean. Bee Culture, 84, (5), 295 à 318.

Lovell H. B., 1958. Let's talk about honey plants, Glean. Bee Culture, 86, (5), 292.

Lovell H. B., 1959. a. Let's talk about honey plants, Glean. Bee Culture, 87, (2), 100-101.

Lovell H. B., 1959. b. Let's talk about honey plants, Glean. Bee Culture, 87, (5), 291-292.

Lovell H. B., 1961. a. Let's talk about honey plants, Glean. Bee Culture, 89, (9), 547-548.

LovelL H. B., 1961. b. Let's talk about honey plants, Glean. Bee Culture, 89, (2), 99-100.

MaUrizio Anna, 1958. 3. Absoluter Gehalt pflanzlicher Bestandteile in Esparsette-, Luzerne-, Orangen-und Rapshonigen. Ann. Abeille, 2, 93-106.

Maurizio Anna, 1949. Pollenanalytische Untersuchungen an Honig und Pollen. A. Beiträge zur quantitativen Pollenanalyse des Honigs. Beih. Schweiz. Bienenztg, 2, (18), 320-421.

Maurizio Anna, Louveaux J., 1962. Method of Pollen Analyses of Honey. Bee World, 43, 122-123.

Maurizio Anna, Louveaux J., 1962. b. Methodik der Honig-Pollenanalyse, Z. Bienenforsch, 6, (4), 115-116.

Maunizio Anna et Louveaux J., 1965. Pollen de plantes mellifères d'Europe. Union des groupements apicoles français, Paris, 1965. 
Niemann A., 1936. Honig auf und am Flußlauf. Prakt. Wegweiser Bienenzüchter, 42, (9), 322-325.

Oertel E., 1939. Honey and Pollen Plants of the United States. U.S. Department of Agriculture, Circular Nr. 554, Washington D.C.

Ordetx Ros G. S., 1952. Flora apicola de la america tropical. Editorial Lex, La Habana, 1952.

Pelletr F. C., 1920. American honey plants, Verlag American Bee Journal, Hamilton.

RahmLow H.J., 1960. Tupelo honey production. Glean. Bee Culture, 88, (8), 509, 1960.

Robinson F. A., 1956. Honey production in central Florida. Glean Bee Culture, 84, (10), 585-589, 637.

SMaLl J. K., 1933. Manual of the southeastern fora. University of North Carolina Press Chapel Hill.

Tsukada M., 1964. Pollen morphology and identification III. Modern and fossil tropical pollen with emphasis on Bombacaceae. Pollen et Spores, 6, 393-462.

VorwoнL G., 1964. a. Die Messung der elektrischen Leitfähigkeit des Honigs und die Verwendung der Messwerte zur Sortendiagnose und zum Nachweis von Verfälschungen mit Zuckerfütterungshoning. Z. Bienforsch., 7, (2), 37-47.

Vorwoнl G., $b$. Die Beziehung zwischen der elektrischen Leitfähigkeit der Honige und ihrer trachtmäßigen Herkunft. Ann. Abeille, 7,(4), 301-309.

Vorwohl G., 1968. Grundzüge einer modernen Pollenbeschreibung im Rahmen der Bienen- und Honigkunde. Z. Bienenforsch., 9, (5), 224-229.

Zander E., 1935, 1937, 1941. Beiträge zur Herkunftsbestimmung bei Honig. Pollengestaltung und Herkunftsbestimmungen bei Blütenhonig. Bd. I Berlin, Verl. Reichsfachgruppe Imker. Bd. II Leipzig, Liedloff, Loth u. Michaelis. Bd. III Leipzig, Liedloff, Loth u. Michaelis.

Anschrift des Verfassers :

Dr. G. Vorwohl

Landesanstalt für Bienenkunde

8 Stuttgart-Hohenheim, BRD.

Emil-Wolffstraße 60. 


\section{TAFEL I}

Aвb. 1. - Honig Nr. 1 : Pollen der Myrica-Gruppe zusammen mit einem Pollen der Rhus vernix-Gr. und einer Pilzspore, $500 \mathrm{x}$.

Aвв. 2. - Honig Nr. 3 : Pollen der Persea-Gruppe zusammen mit Ilex und Pollen der Serenoa-Gruppe, $500 \mathrm{x}$.

Авв. 3. - Honig Nr. 9 : Pollen der Piperaceen/Saururaceen-Form zusammen mit Ilex, $500 \mathrm{x}$.

Aвв. 4. - Honig Nr. 7 : Pollen von Itea virginica zusammen mit Citrus, 500 x.

\section{PLANCHE I}

Fig. 1. - Miel no 1 : Pollen du groupe Myrica, avec un pollen du groupe Rhus vernix et une spore de champignon, $500 \mathrm{x}$.

Fig. 2. - Miel no 3 : Pollen du groupe Persea, avec Ilex et pollen du groupe Serenoa, $500 \mathrm{x}$.

Fig. 3. - Miel no 9 : Pollen du type Piperaceae/Saururaceae, avec Ilex, 500 x. FIg. 4. - Miel no 7 : Pollen d'Itea virginica, avec Citrus, 500 x. 


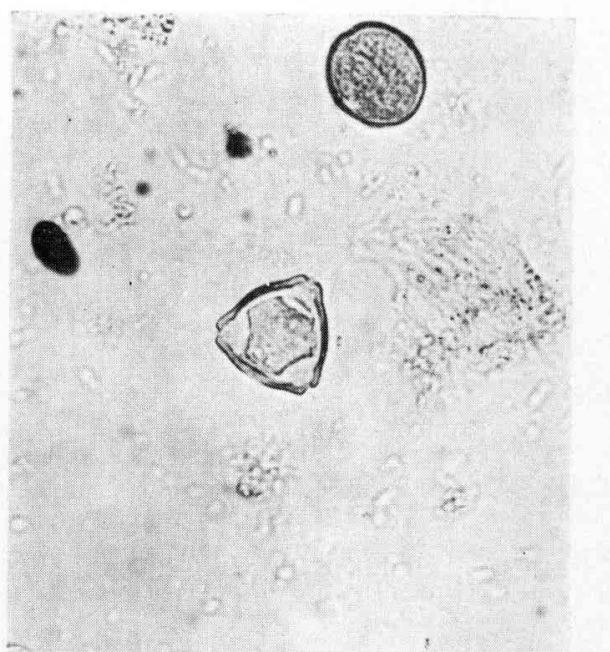

Fig. I
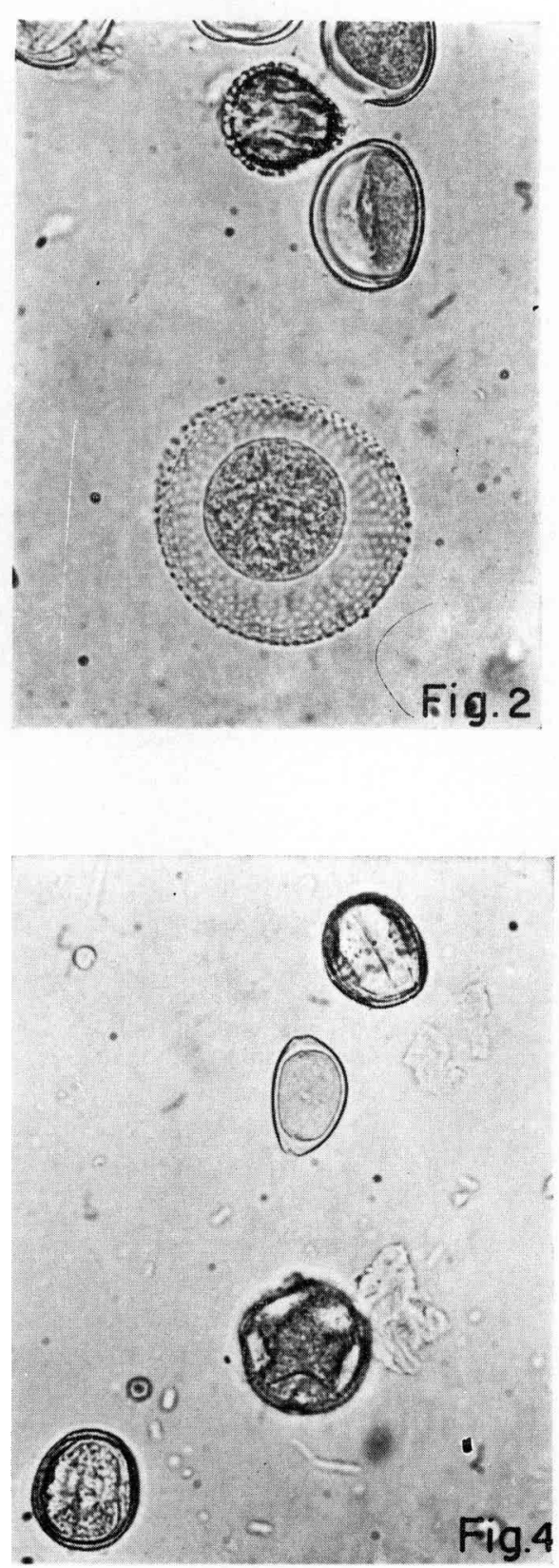

G. VORWOHL 
TAFEL II

Aвв. 5. - Honig Nr. 3 : Mimosaceen-Tetrade mit Pollen der Serenoa - und Sophora - Gruppe, $500 \mathrm{x}$.

Авв. 6. - Honig Nr. 2 : Pollen von Bursera zusammen mit Pollen der SerenoaGruppe. Am Oberrand Pollen der Bidens-Gr., 500 x.

\section{PLANCHE II}

Fig. 5. - Miel $n^{0} 3$ : Tetrade de Mimosaceae, avec pollens des groupes Serenou et Sophora, $500 \mathrm{x}$.

Fig. 6. - Miel no 2 : Pollen de Bursera, avec pollen du groupe Serenoa. Sur le bord supérieur, pollen du groupe Bidens, $500 \mathrm{x}$. 
Apidologie, 1970, 1 (3).

Pl. II
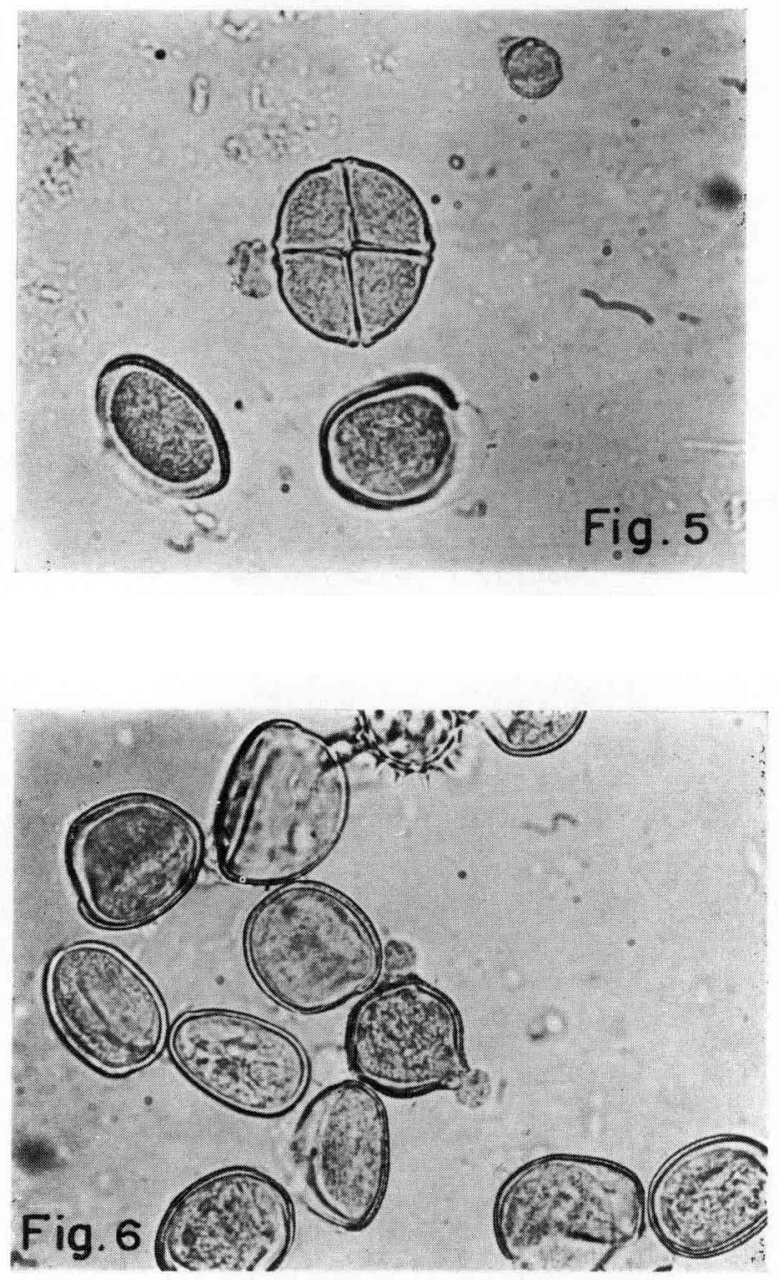

G. VORWOHL 
Aвв. 7. - Honig, Nr. 11 : a. Pollen des Pistacia-Typs zusammen mit Nyssa und Pollen der Rhus vernix-Gruppe, 500 x. - b. Pollen des Pistacia-Typs, 5seitig ausgequollen, zusammen mit $N y s s a$, sowie Pilzhyphen und Sporen, 500 x. - c. Pollen des Pistacia-Typs, hier mit nur 4. Keimstellen, ferner Nyssa, $500 \mathrm{x}$.

\section{PLANCHE III}

Fig. 7. - Miel no $11: a$. Pollen du type Pistacia, avec Nyssa, et pollen du groupe Rhus vernix, $500 \mathrm{x} .-b$. Pollen du type Pistacia à 5 sillons, avec Nyssa, ainsi que des hyphes et des spores de champignons, $500 \times$ x. $-c$. Pollen du type Pistacia, avec ici seulement 4 sillons, plus loin Nyssa, $500 \mathrm{x}$. 
Apidologie, 1970, 1 (3).

PI. III
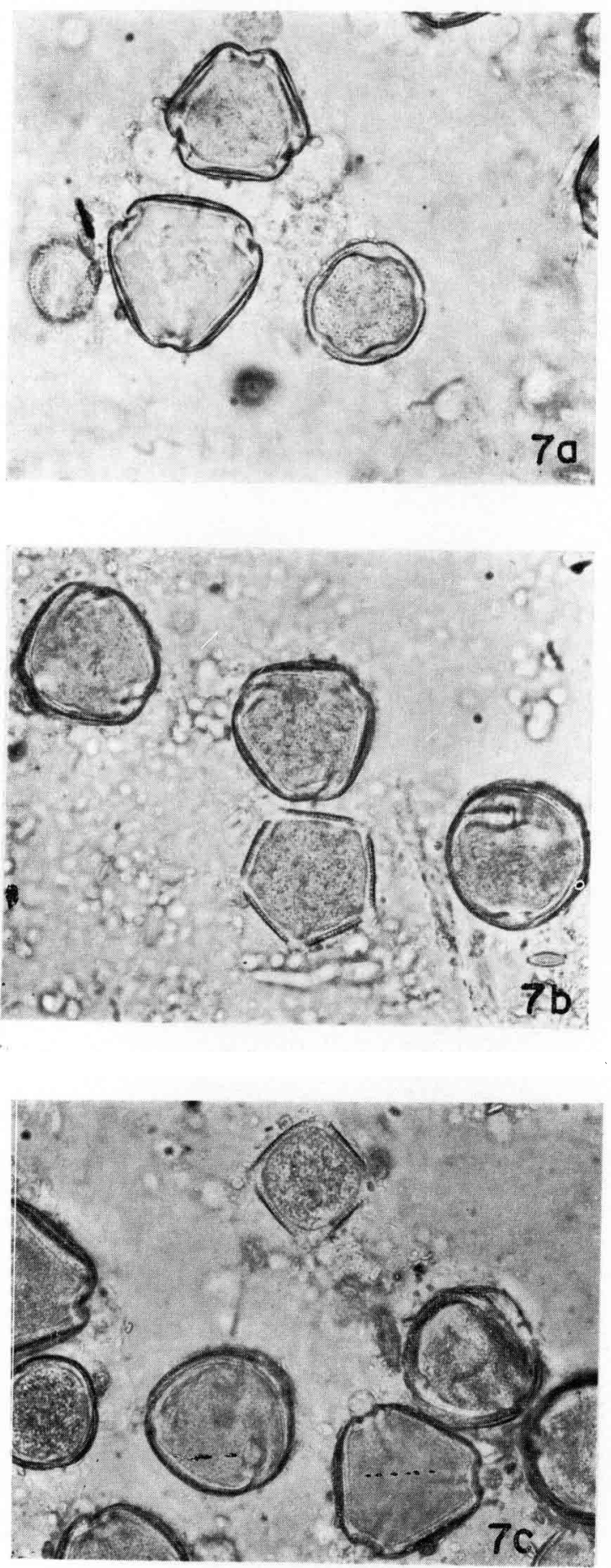

G. VORWOHL 
Авв. 8. - Honig N1. 5 : Pollen von Sambucus (S. nigra-Gruppe) zusammen mit Citrus und Quercus, $500 \mathrm{x}$.

Авв. 9. - Carya tomentosa : a. Pollen in der Pollage, optischer äquatorialer Schnitt, $1000-\mathrm{x}$. b. Pollen in der Seitenlage, meridionaler optischer Schnitt, $1.000 \mathrm{x}$.

\section{PLANCHE IV}

Fig. 8. - Miel no 5 : Pollen de Sambucus (groupe S. nigra), avec Citrus et Quercus, $500 \mathrm{x}$.

Fig. 9. - Carya tomentosa : a. Pollen en vue polaire, coupe optique équatoriale, 1.000 x. - b. Pollen en vue équatoriale, coupe optique méridienne, $1.000 \mathrm{x}$. 
Apidologie, 1970, 1 (3).

Pl. IV
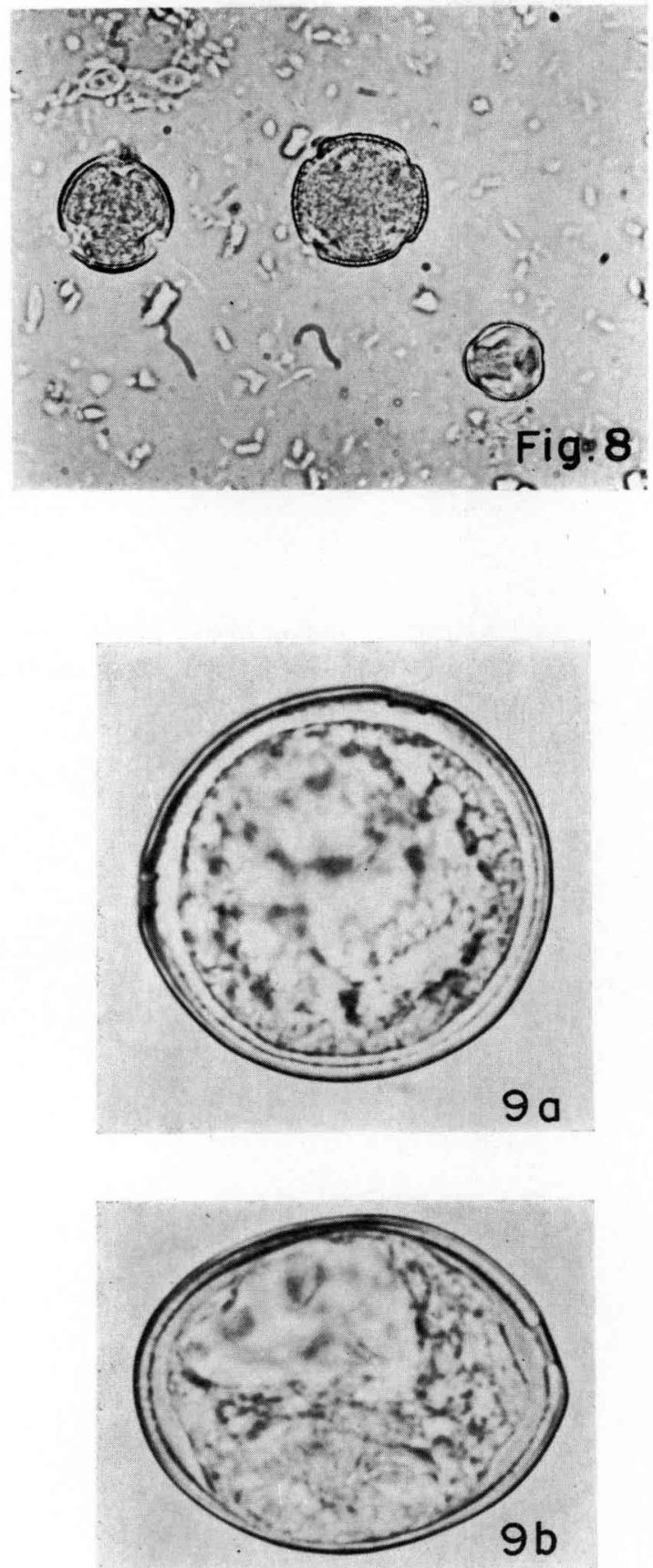

G. VORWOHL 
Aвb. 10. - Gordonia lasianthus : a. Pollen in der Pollage, Focus 1, 1.000 x. $b$. Pollen in der Pollage, optischer äquatorialer Schnitt, $1.000 \mathrm{x}$ - $c$. Pollen in der Seitenlage, Focus 2, 1.000 x. $-d$. Pollen in der Seitenlage, optischer meridionaler Schnitt, $1.000 \mathrm{x}$.

\section{PLANCHE V}

Fig. 10. - Gordonia lasianthus : a. Pollen en vue polaire, Focus 1, 1.000 x. $b$. Pollen en vue polaire, coupe optique équatoriale, $1.000 \mathrm{x}$. $-c$. Pollen en vue équatoriale, Focus 2, 1.000 x. - $d$. Pollen en vue équatoriale, coupe optique méridienne, $1.000 \mathrm{x}$. 
Apidologie, 70, 1 (3).

Pl. V
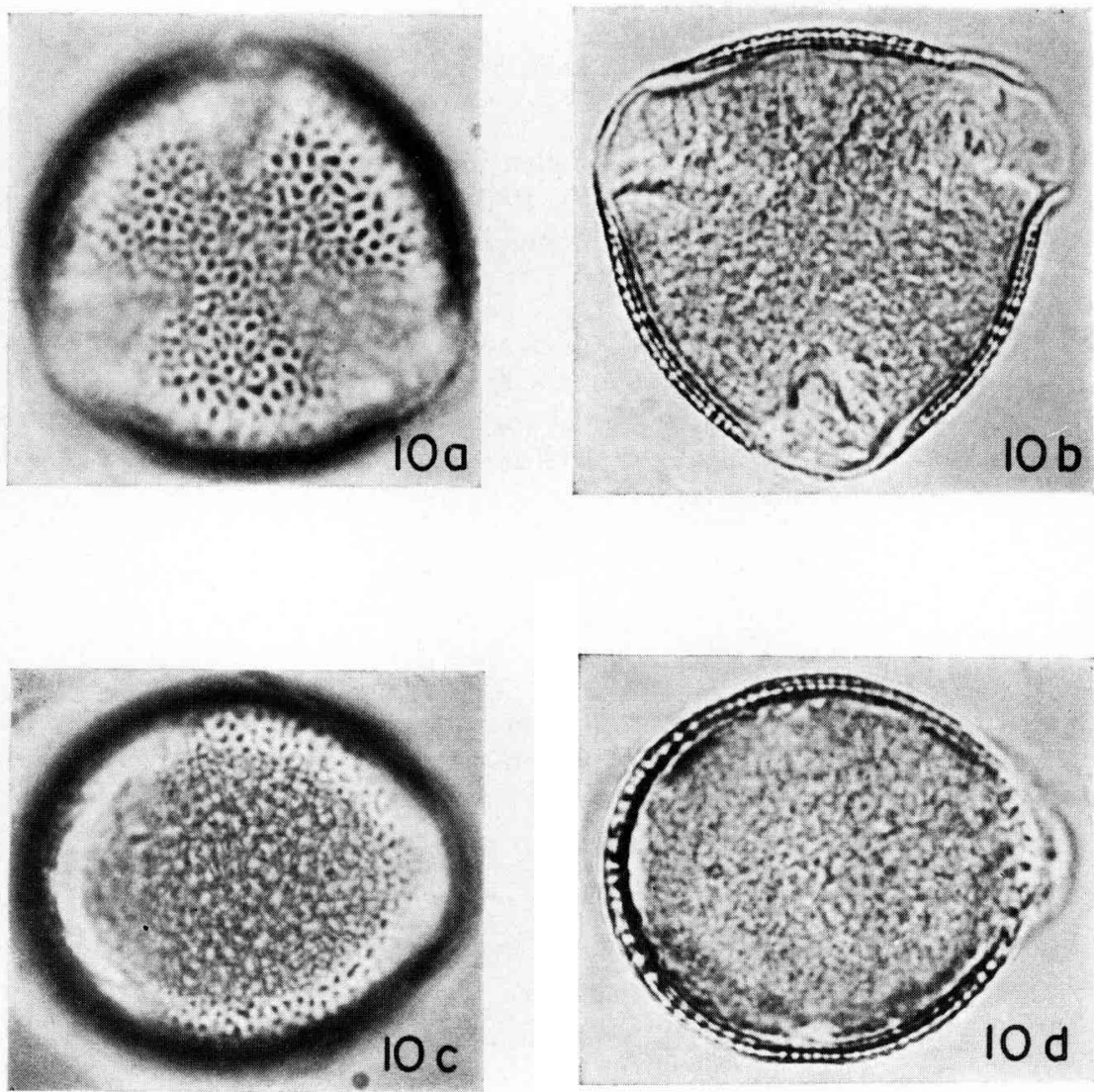

G. VORWOHL 


\section{TAFEL VI}

Aвв. 11. - Fagara rhoifolium : a. Pollen in der Seitentage, Focus 1, Aufsicht auf das Intercolpium, $1.000 \times$ x. $-b$. Pollen in der Seitenlage, optischer meridionaler Schnitt, $1.000 \mathrm{x} .-c$. Pollen in der Pollage, optischer äquatorialer Schnitt, $1.000 \mathrm{x}$.

Aвв. 12. - Melia azedarach : a. Pollen in der Pollage, optischer äquatorialer Schnitt, 1.000 x. $-b$. Pollen in der Seitenlage, optischer meridionaler Schnitt, 1.000 x. - c. Pollen in der Seitenlage, Aufsicht auf die Porenfalte, Focus 1, 1.000 x. $-d$. Pollen in der Seitenlage, Aufsicht auf ein Intercolpium und zwei Porenfalten, Focus 1, $1.000 \mathrm{x}$.

\section{PLANCHE VI}

Fig. 11. - Fagara rhoifolium : a. Pollen en vue équatoriale, Focus 1, vue de l'Intercolpium, $1.000 \times$. $-b$. Pollen en vue équatoriale, coupe optique méridienne, $1.000 \mathrm{x} .-c$. Pollen en vue polaire, coupe optique équatoriale $1.000 \mathrm{x}$.

FIg. 12. - Melia azedarach : a. Pollen en vue polaire, coupe optique équatoriale, $1.000 \mathrm{x} .-b$. Pollen en vue équatoriale, coupe optique méridienne, 1.000 x. - c. Pollen en vue équatoriale, vue des colporus, Focus 1, 1.000x.$d$. Pollenen vue équatoriale, vue d'un intercolpium et de deux colporus, Focus 1,1.000 x. 
Apidologie, 1970, 1 (3).

P1. VI
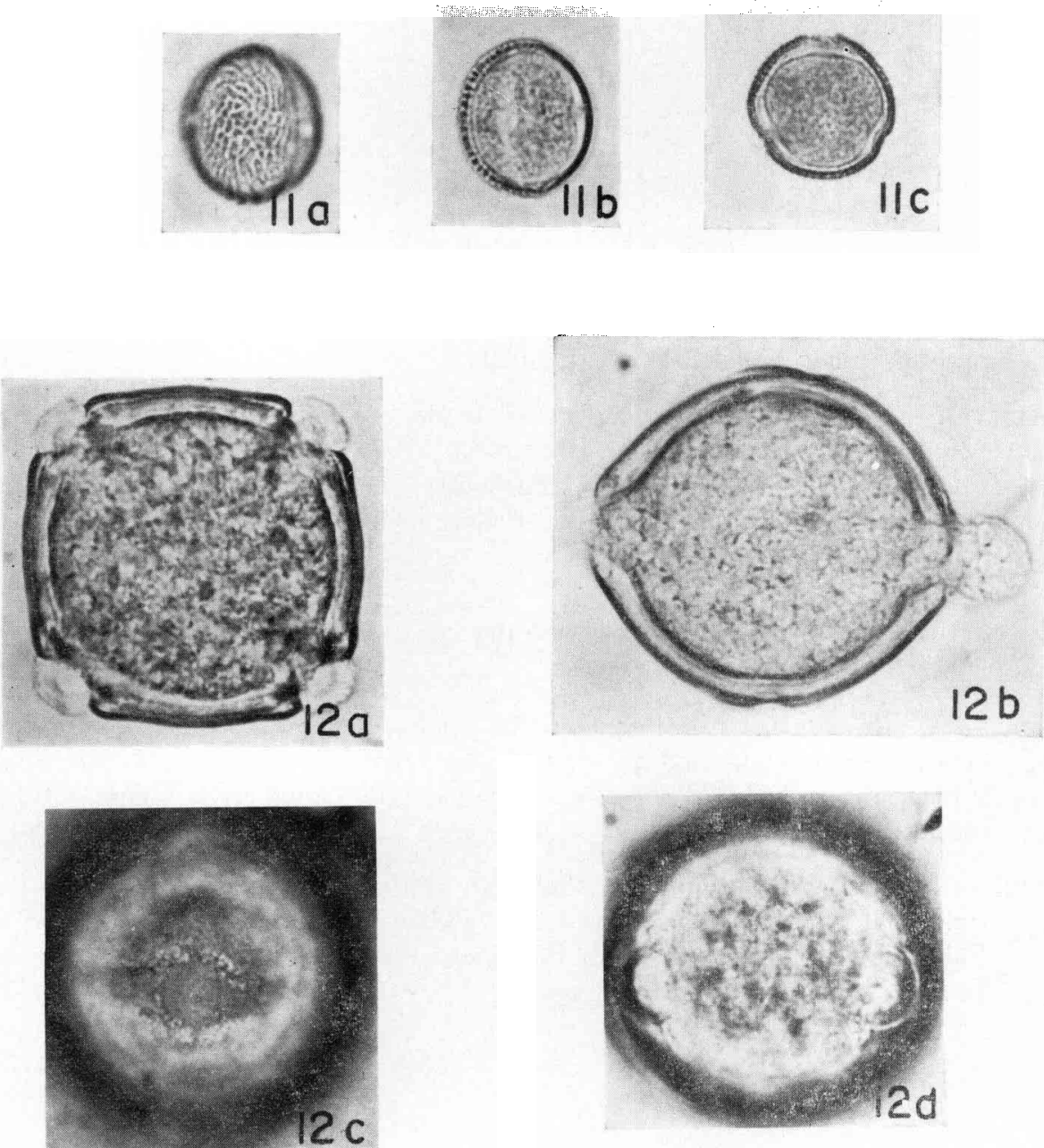

G. VORWOHL 
Aвв. 13. - Rhus vernix : a. Pollen in der Pollage, Aufsicht, Focus 1, 1.000x.$b$. Pollen in der Pollage, optischer äquatorialer Schnitt, $1.000 \mathrm{x}$. $-c$. Pollen in der Seitenlage, Aufsicht, Focus 1, 1.000 x. $-d$. Pollen in der Seitenlage, optischer meridionaler Schnitt, $1.000 \mathrm{x}$.

Aвв. 14. - Richardia scabra : a. Pollen in der Pollage, optischer äquatorialer Schnitt, $500 \mathrm{x}$. Pollen ungefärbt. - $b$. Pollen in der Pollage, Aufsicht, Focus 2, $500 \mathrm{x}$, Pollen gefärbt (Fuchsin). - $c$. Pollen in der Seitenlage, Aufsicht auf die Falten, Focus 1, Pollen gefärbt, $500 \mathrm{x}$.

\section{PLANCHE VII}

Fig. 13. - Rhus vernix : $a$. Pollen en vue polaire, Focus $1,1.000$ x. $-b$. Pollen en vue polaire, coupe optique équatoriale, $1.000 \times$ x. $-c$. Pollen en vue équatoriale, Focus 1, $1.000 \mathrm{x}$ - - $d$. Pollen en vue équatoriale, coupe optique méridienne, $1.000 \mathrm{x}$.

Fig. 14. - Richardia scabra : $a$. Pollen en vue polaire, coupe optique équatoriale, $500 \mathrm{x}$. Pollen non coloré. - $b$. Pollen en vue polaire, Focus $2,500 \mathrm{x}$, Pollen coloré (Fuchsine). $-c$. Pollen en vue équatoriale, vue des sillons, Focus 1, Pollen coloré, $500 \mathrm{x}$. 

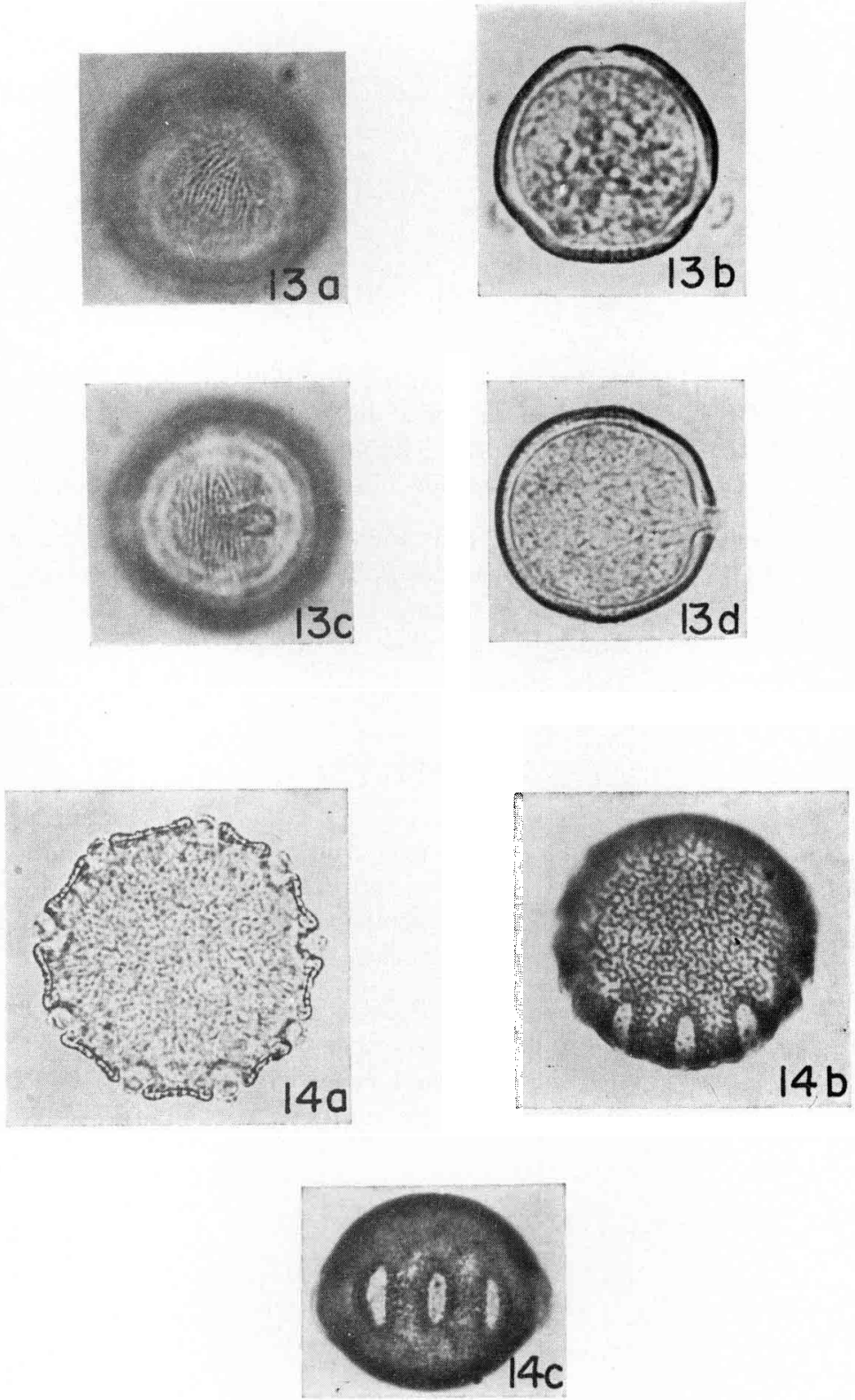

G. VORWOHL 
TAFEL VIII

Aвв. 15. - Bidens bipinnata : a. Pollen in der Pollage, Aufsicht auf das Polfeld, 1.000 x. - b. Pollen in der Pollage, optischer äquatorialer Schnitt, 1.000 x. - c. Pollen in der Seitenlage, Aufsicht auf die Porenfalte, $1.000 \mathrm{x} .-d$. Pollen in der Seitenlage, optischer meridionaler Schnitt, $1.000 \mathrm{x}$.

Авв. 16. - Helenium tenuifolium : a. Pollen in der Pollage, Aufsicht auf das Polfeld, 1.000 x. $-b$. Pollen in der Pollage, optischer äquatorialer Schnitt, $1.000 \times$ x. $-c$. Pollen in der Seitenlage, Aufsicht auf die Porenfalte, $1.000 \mathrm{x} .-$ $d$. Pollen in der Seitenlage, optischer meridionaler Schnitt, $1.000 \mathrm{x}$.

\section{PLANCHE VIII}

Fig. 15. - Bidens bipinnata ; $a$. Pollen en vue polaire, vue du champ polaire, $1.000 \mathrm{x} .-b$. Pollen en vue polaire, coupe optique équatoriale, $1.000 \mathrm{x} .-$ $c$. Pollen en vue équatoriale, vue des colporus, $1.000 \mathrm{x}$. $-d$. Pollen en vue équatoriale, coupe optique méridionale, $1.000 \mathrm{x}$.

Fig. 16. - Helenium tenuifolium : a. Pollen en vue polaire, vue du champ polaire, $1.000 \mathrm{x} .-b$. Pollen en vue polaire, coupe optique équatoriale, 1.000 x., $-c$. Pollen en vue équatoriale, vue des colporus, $1.000 \mathrm{x}$. $d$. Pollen en vue équatoriale, coupe optique méridienne, $1.000 \mathrm{x}$. 
Apidologie, 1970, 1 (3).

P1. VIIl
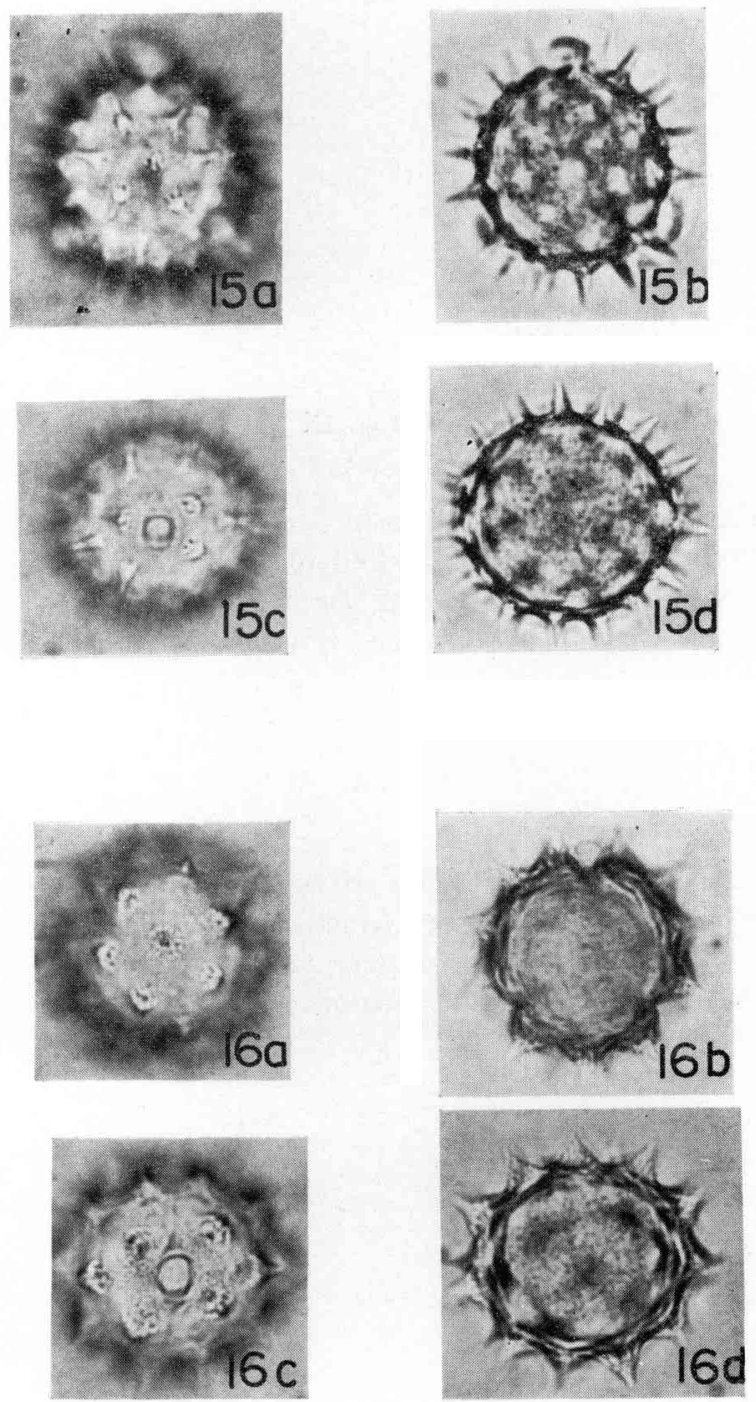

G. VORWOHL 
Aвв. 17. - Sabal palmetto : a. Pollen in der Seitenlage, optischer meridionaler Schnitt, 1.000 x. - b. Pollen in der Seitenlage, Aufsicht auf das Intercolpium, Focus 1, 1.000 x. - c. Pollen in der Seitenlage, Aufsicht auf die Falte, 1.000 x. $-d$. Pollen in der Pollage, optischer äquatorialer Schnitt, 1.000 x.

\section{PLANCHE IX}

Fig. 17. - Sabal palmetto : a. Pollen en vue équatoriale, coupe optique méridienne, $1.000 \times$. $-b$. Pollen en vue équatoriale, vue de l'intercolpium, Focus 1 , $1.000 \mathrm{x} .-c$. Pollen en vue équatoriale, vue des sillons, $1.000 \mathrm{x}$. $-d$. Pollen en vue polaire, coupe optique équatoriale, $1.000 \mathrm{x}$. 

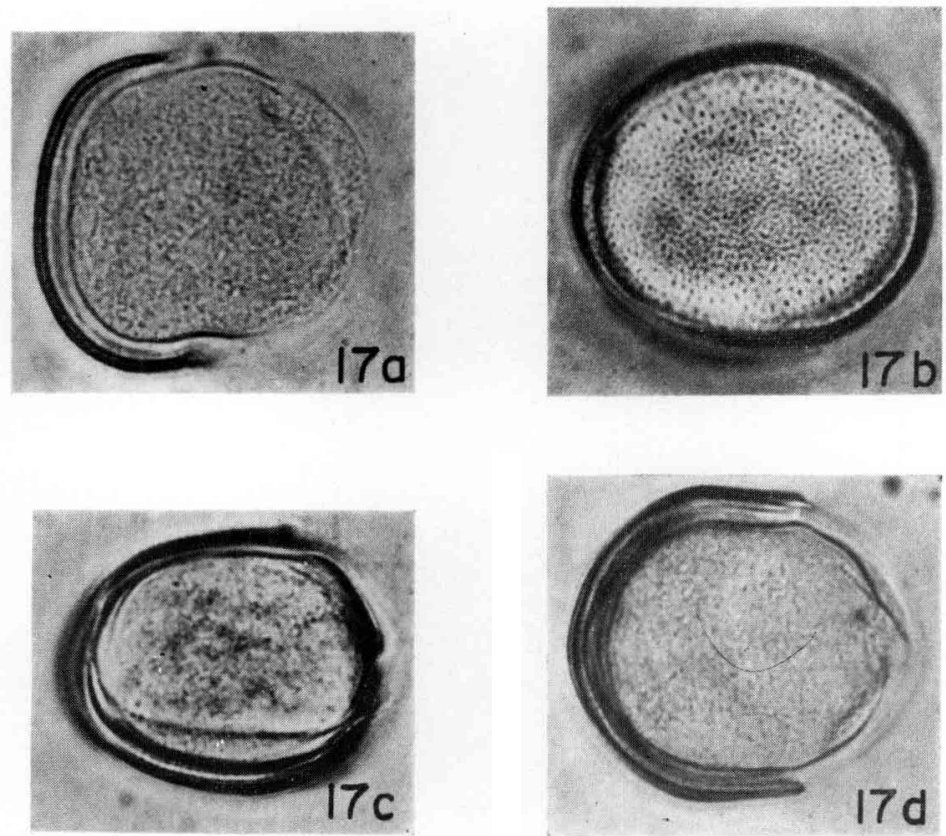

G. VORWOHL 